\title{
Bending Characteristics of Foldable Touch Display Panel with a Protection Structure Design
}

\author{
Hsien-Chie Cheng, ${ }^{1}$ Wei-Heng Xu, ${ }^{2}$ Wen-Hwa Chen, ${ }^{2}$ Pi-Hsien Wang, \\ Kuo-Feng Chen, ${ }^{3}$ and Chih-Chia Chang ${ }^{3}$ \\ ${ }^{1}$ Department of Aerospace and Systems Engineering, Feng Chia University, Taichung 40724, Taiwan \\ ${ }^{2}$ Department of Power Mechanical Engineering, National Tsing Hua University, Hsinchu 30013, Taiwan \\ ${ }^{3}$ Display Technology Center, Industrial Technology Research Institute, Chutung 31040, Taiwan
}

Correspondence should be addressed to Hsien-Chie Cheng; hccheng@fcu.edu.tw and Wen-Hwa Chen; whchen@pme.nthu.edu.tw

Received 22 September 2014; Revised 8 March 2015; Accepted 10 March 2015

Academic Editor: Zoe Barber

Copyright (C) 2015 Hsien-Chie Cheng et al. This is an open access article distributed under the Creative Commons Attribution License, which permits unrestricted use, distribution, and reproduction in any medium, provided the original work is properly cited.

\begin{abstract}
The study proposes and demonstrates an enhancement of a touch display panel (TDP) through a polymer-based protection structure to achieve higher bendability and reliability. The bending performance of the TDP without or with the protection structure designs is addressed using three-dimensional geometry-nonlinear finite element analysis and mechanical testing. The elastic properties of the components in the TDP structure are derived from nanoindentation and uniaxial tensile/compressive testing. The calculated results are compared with each other and also against the experimental bending fatigue test data. At last, a design guideline and optimal factor setting for enhanced bending performance are sought through parametric FE analysis and Taguchi experimental design, respectively. The optimal design is compared with the original in terms of bending stress. The simulation results show that bending would create significant tensile and compressive bending stresses on the indium tin oxide/dielectric layers, which are the main cause of several commonly observed failures, such as thin film cracking and delamination, in a thin rigid film coating on a thick compliant substrate. It also turns out that a substrate with a lower stiffness has a better mechanical stability against bending stress.
\end{abstract}

\section{Introduction}

Flexible electronics is an emerging and rapid-developing technology that fabricates thin electronic components and devices on a rollable, bendable, stretchable, foldable, or flexible substrate. It functions beyond the capability of rigid electronics products, offering great advantages, such as small form factor, cost effectiveness, lightweight, unbreakability, flexibility, and portability. The technology is gaining more and more attention worldwide because it is the promising future of electronics products with great potential for use in many electronics applications, such as flexible display, flexible touch panel, wearable electronic devices, biomedical sensors, and printed batteries. Over the last decades, extensive studies have been made on the research and development of flexible displays and foldable touch panels. Flexible display with foldable touch panel, briefly termed flexible touch display panel (TDP), promises to become the mainstream of nextgeneration portable applications and display systems.

Flexible TDP is required to be repeatedly and severely bended, flexed, or folded during service. In the literature, some work has been done on the bending behaviors and performance of flexible TDP. For example, an $\left(\mathrm{In}_{2} \mathrm{O}_{3}\right)_{100-x}$ $(\mathrm{ZnO})_{x}(\mathrm{IZO})$ film on a polyethylene terephthalate (PET) substrate with $\mathrm{SiO}_{2}$ buffer layers by the rf-magnetron sputtering was proposed by Park et al. [1] for achieving a transparent conducting oxide film for flexible displays. Compared to indium tin oxide (ITO) films, IZO films show superior electrical conductivity and electrical stability due to the bending stress. Sim et al. [2] found that a thin metal buffer layer with a minimized loss of transparency can highly upgrade the mechanical stability of ITO film deposited on the plastic substrate. Alzoubi et al. [3] conducted high cycle bending fatigue experiments on an ITO coated PET substrate, 


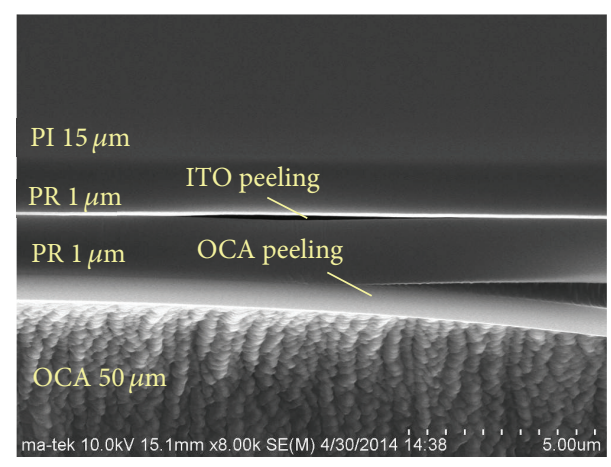

(a) Peeling at the ITO and OCA interfaces under a tensile stress

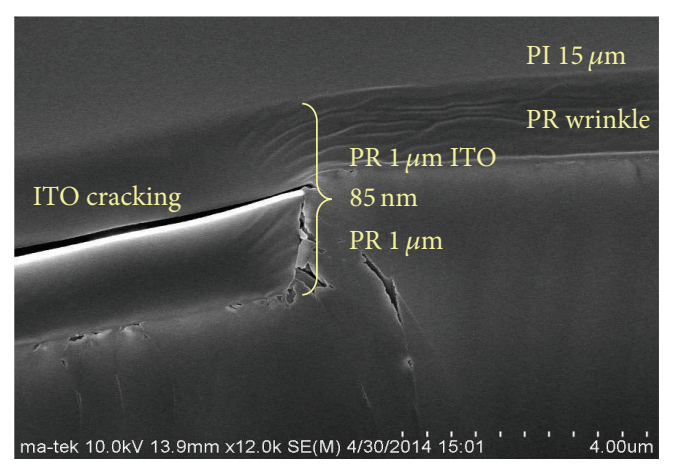

(b) Buckling delamination under a compressive stress

FIGURE 1: Failure mechanisms of a TDP structure under bending.

where the effects of bending diameter, bending frequency, and sample width on the change in electrical resistance were investigated. It was found that bending diameters as well as the number of bending cycles have a great influence on the electrical conductivity of the ITO layer. Caims et al. [4] reported on the mechanical and electrical reliability of an ITO coating on a PET substrate. They found that the mechanical behavior of the ITO film is dominated by the properties of the substrate, and the mechanical reliability of the ITO layer is greatly influenced by the film thickness. Oh et al. [5] investigated the failure mechanism of patterned ITO electrodes on a PET substrate under static and cyclic bending in the mechanical stress mode. They found that two types of microcracks are observed depending on the stress modes. To improve electrical and optical properties under static and dynamic mechanical bending, Yu et al. [6] deposited ITO films on PET substrates using a thermionic emission (TE) enhanced DC magnetron sputtering system.

Though great effort and progress have been accomplished in realizing a bendable, flexible, and foldable TDP, there remains a great challenge in achieving a very large bending curvature. Basically, a TDP structure is constructed by stacking multiple thin composite films of different materials, thicknesses, and even sizes. The multilayered structure tends to have different thermal-mechanical properties, such as coefficient of thermal expansion, Poisson's ratio, modulus, and strength. For example, ITO is most commonly applied as a conductive and electrode material because of its many features that are needed for building flexible touch display panels, including transparency, good electrical properties, and ease of fabrication. Unfortunately, ITO is brittle and hard in nature and would crack at a very small tensile strain, thereby causing failure in the conductive layer. In addition, the material properties of the transparent conductive oxide are radically distinct from those of the other intimately bonded materials in the multilayered structure, such as dielectric layers and substrate and adhesive layer. The thin multilayered structure, though having a better bendability and lighter weight, would suffer from structure stability problems and being more susceptible to fatigue. For instance, excessive bending would create a high tensile and compressive stress in the multilayered structure due to the mismatch of material properties and large deformation, probably leading to thin film cracking (see, e.g., $[7,8]$ ) and delamination, as shown in Figure 1(a). Furthermore, the compressive stress tends to result in interfacial delamination as a result of local buckling, as shown in Figure 1(b), commonly observed in the interface of a thin rigid film stacked on a thick compliant substrate. The buckling delamination $[7,9-13]$ is one of the most susceptible failure mechanisms in flexible displays and TDP structure. For example, Kardomateas [9] experimentally and theoretically explored the snap buckling phenomenon of a delaminated composite under pure bending at certain conditions of applied bending load and geometrical configuration. Cotterell and Chen [11] studied the buckling and cracking behavior of thin films on compliant substrates under compression. They found that the energy stored in the compliant substrate greatly contributes to the energy release rate of the rigid film delamination under compression either with or without cracking unless the substrate is at least as stiff as the film. Yu and Hutchinson [12] investigated a straight-sided delamination buckle of a thin film deposited on a substrate under in-plane compressive stress with a focus on the effects of substrate compliance. They found that the more compliant the substrate, the easier for the film to buckle and the higher the energy release rate. The effect becomes significant when the modulus of the substrate is appreciably less than that of the film. Lim et al. [10] experimentally reported the mechanical and electrical performance of various ITO coated polymer substrates under bending. They found that the mechanical and electrical performance of the ITO coated polymer substrate under continuous bending stress condition would be strongly dependent on the material properties of base polymer material such as Young's modulus, where a polymer substrate with a lower Young's modulus would hold better mechanical and electrical stability against bending stresses.

The cohesive and interfacial failures would limit the bendability and mechanical stability of the flexible TDP structure (Figure 2). Bendability and mechanical stability [10, 14] are two of the most critical issues facing the technology today. Prior to successful implementation of the flexible TDP technology, there is a crucial need of clearly understanding 


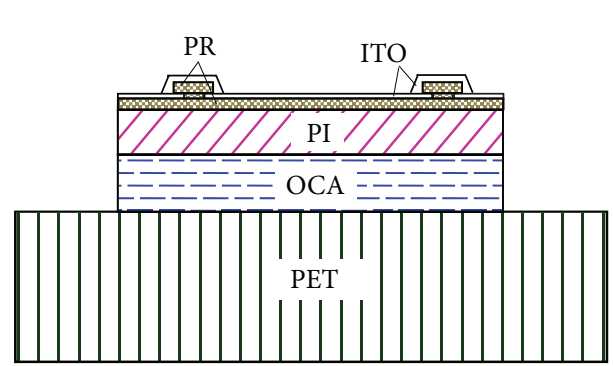

(a) Without protection structure

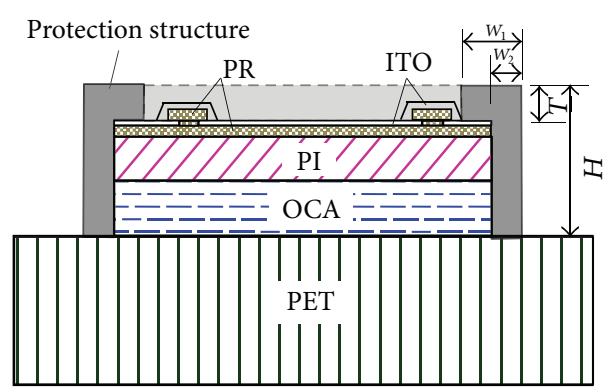

(b) With protection structure

FIGURE 2: The TDP structure with or without a protection structure.

the bending behavior and fully minimizing the bending stress so as to enhance its bendability and reliability. In the study, an enhancement of the bendability and mechanical stability of a TDP structure is proposed and demonstrated through a polymer-based protection structure design, as shown in Figure 2(b). The protection structure is used to protect the touch panel laminate structure from bendinginduced fatigue. Two different protection structure designs are introduced: (1) the full protection structure (FPS) design and (2) the side protection structure (SPS) design. The bending characteristics of the TDP with or without the protection structure designs under a four-point bending load are explored through both three-dimensional (3D) geometrynonlinear FE analysis and mechanical testing. Nanoindentation and uniaxial tensile/compressive testing are carried out to assess the elastic properties of the components in the TDP structure. The calculated results are compared with each other and also those of the no protection structure (NPS) design, as shown in Figure 2(a). Furthermore, the effects of the geometry and material properties of the protection structure and touch panel laminate on the bending mechanical behavior are examined through parametric FE analysis. Finally, Taguchi experimental design incorporated with the 3D nonlinear FE modeling is carried out to seek the optimal factor setting for better bending performance.

\section{TDP Laminate Structure}

A schematic of the cross section of the multilayered TDP structure with and without the protection structure design considered in the investigation is shown in Figure 2. The TSP mainly consists of a display panel module for displaying images, a touch panel module for sensing touch input, and an adhesion unit for mechanically and electrically bonding the display panel module and the touch panel module. Specifically, it comprises seven thin film layers, namely, two ITO conductive layers of $0.07 \mu \mathrm{m}$ thickness, two organic photoresist (PR) dielectrics of $1 \mu \mathrm{m}$ thickness, a transparent polyimide (PI) substrate of $15 \mu \mathrm{m}$ thickness for touch panel (briefly termed touch panel substrate), an optically clear adhesive (OCA) of $20 \mu \mathrm{m}$ thickness, and a PET substrate of $100 \mu \mathrm{m}$ thickness for display panel (briefly termed display panel substrate). The dimension of the TDP is $12.0 \times 9.0\left(\mathrm{~cm}^{2}\right)$. The protection structure is made of a polymer material. In the investigation, two different types of protection structure designs are considered, as shown in Figure 2(b). The first one is a full protection structure (FPS) design, where the entire surfaces of the touch panel laminate structure exposed to ambient environment, namely, the top and periphery, are overcoated with a polymer material. It is noted that a full cover of the touch screen panel by a thin layer of the polymer material, just as the FPS design, may affect its optical transparency, form factor, and even flexibility. Alternatively, a side protection structure (SPS) design is also introduced, featuring only a partial cover of the touch screen panel, as shown in Figure 2(b), where the geometric parameters are as follows: $W_{1}=800 \mu \mathrm{m}, W_{2}=$ $400 \mu \mathrm{m}, H=50 \mu \mathrm{m}$, and $T=12.86 \mu \mathrm{m}$. In the study, the TDP without a protection structure is termed the original design or alternatively the no protection structure (NPS) design.

\section{FE Modeling}

In the study, in addition to experimental testing, a 3D nonlinear FE modeling is applied for characterizing the bending characteristics of the flexible TDP structure under four-point bending load. As mentioned earlier, the dimension of the SPS/FPS type TDP is $12.0 \times 9.0 \times 0.015\left(\mathrm{~cm}^{3}\right)$, which is composed of seven very thin layers. Noticeably, these thin layers and even the TSP structure possess a very poor aspect ratio. Comprehensively performing detailed FE modeling of the laminate structure would end up with an enormous mesh size and also require a great computational and modeling effort. There is a huge and persistent favor of avoiding timeconsuming FE modeling and computation. Thus, in the investigation, instead of using the full TSP model, a small nominal TSP of the size $1.0 \times 1.0 \times 0.015\left(\mathrm{~cm}^{3}\right)$ is applied. The modeling is later on demonstrated to be adequate and effective for qualitative evaluation and comparison of the bending performance of the TSP structures with or without a protection structure design. Besides, the dielectric and conductive layers contain patterned PR materials and ITO routing traces, which consist of not only a very poor aspect ratio but also a very complicated geometry and multiple material constituents (e.g., PR and ITO). For further modeling simplification and computational cost reduction, macroscopic constitutive modeling is applied for those patterned 


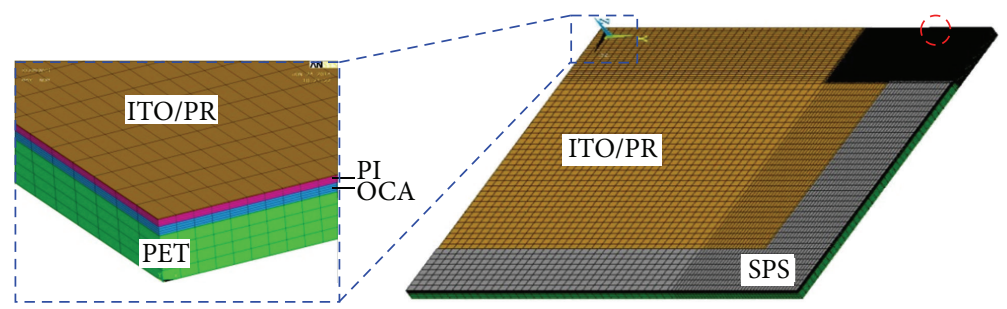

FIGURE 3: 3D FE model of the flexible TDP structure with a side protection structure (SPS).
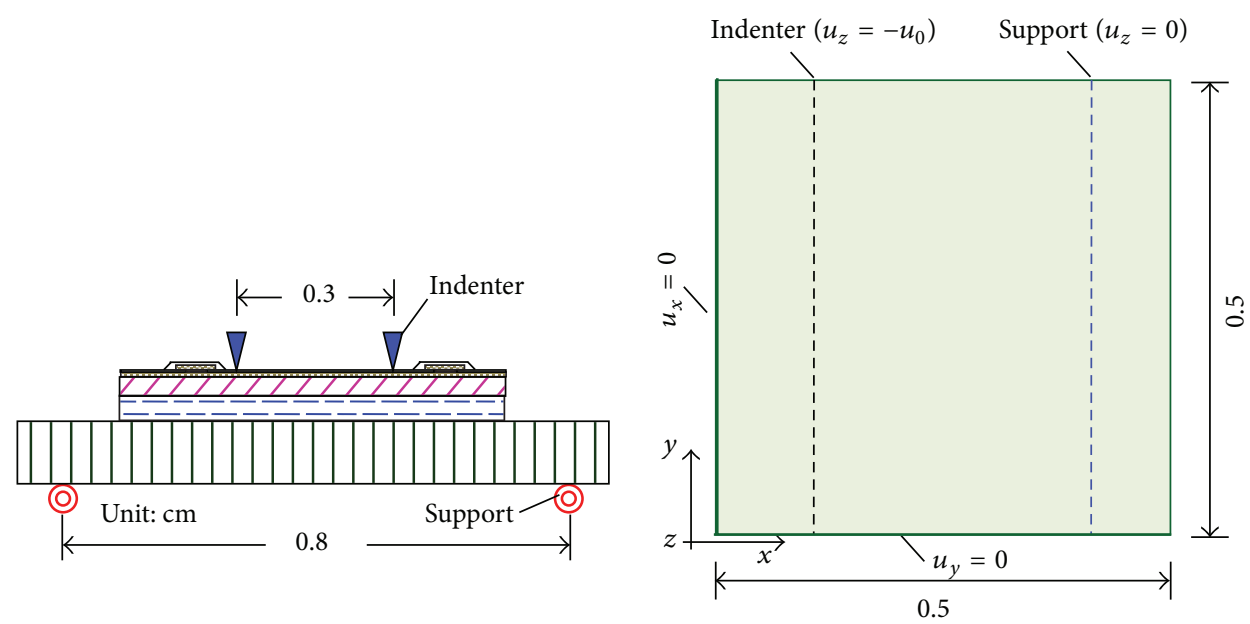

FIGURE 4: A schematic of four-point bending test and associated boundary condition for FE modeling.

layers. In other words, they are modeled as an isotropic, bulk material, where effective Young's modulus and Poisson's ratio are evaluated by an average approach. In the literature, various averaging approaches have been proposed, such as rule of mixture (ROM) technique [8], FE-based averaging technique $[15,16]$, homogenization method [17], and selfconsistent method [18]. In the study, we make use of an ROM approach due to its effectiveness and efficiency. The volume fraction of the first dielectric and conductive layers is only about $0.4 \%$ and $0.1 \%$ of the corresponding layer volume, respectively. Accordingly, they are not taken into account in the present FE modeling. On the other hand, the macroscopic constitutive modeling is applied on the second conductive layer, which takes a volume fraction of about $95 \%$. Because of such a high volume fraction, the loss of prediction accuracy due to modeling simplification would be very minimal.

In order to further minimize the computational effort, the 3D FE modeling creates a local-fine-mesh at the region where the stress and strain are extracted and a coarse mesh elsewhere. An example of the constructed 3D local-finemesh FE model, which is for the flexible TDP structure with the SPS design, is shown in Figure 3, consisting of 284000 elements and 308240 nodes. The extraction site is also shown in a dotted circle in Figure 3 for both with or without protection structure, which is very close to the symmetry boundary. The flexible TDP structure with or without the protection structure design that is considered as the test vehicle of the study consists of a square shape with a side length of $1 \mathrm{~cm}$.
Due to symmetry, only a quadrant of the flexible TDP is considered in the modeling where a symmetry boundary condition is imposed on the corresponding symmetry planes. Geometry nonlinearity that takes into account large deformation and rigid body motion is also considered. Young's modulus and Poisson's ratio of the second ITO layer are 106.5 GPa and 0.475 ; by using the ROM technique, equivalent Young's modulus and Poisson's ratio are $101.7 \mathrm{GPa}$ and 0.45 , respectively. In addition, Young's modulus of the components in the laminated structure is derived from nanoindentation and uniaxial tensile/compressive testing and also the literature. Some basic assumptions are made in the FE analysis, including perfect adhesion at all the material interfaces and no observed voids and delamination in the structure. To simulate the four-point bending load, as shown in Figure 4, the following boundary conditions are specified, in addition to the aforementioned symmetric conditions. The $z$-dir. degree of freedom, that is, $u_{z}$, of the nodes along the support is constrained while an enforced $z$-dir. displacement is applied on the nodes along the indenter. Note that the TDP structure is bent with a radius of curvature of $0.75 \mathrm{~cm}$.

\section{Taguchi Method}

Taguchi method (see, e.g., $[19,20]$ ) is mainly used for identifying important control factors most affecting the selected objective, such as product quality or performance, and more importantly improving the selected objective. In addition to 


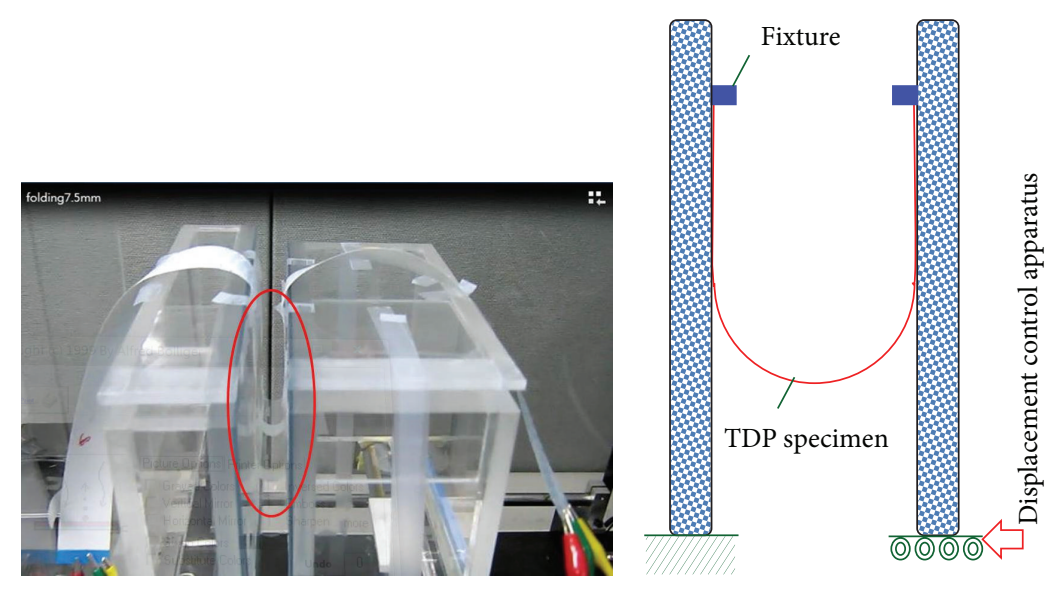

FIGURE 5: Two-point bending tester and test setup.

effectiveness, robustness, and simplicity, this method is also known for its capability of acquiring maximum information with minimum experimental run and thus is widely applied in many industries, such as manufacturing industry and mechanical component design, for enhancing the quality of products or the performance of process. In order to obtain sufficient information with a minimal effort, orthogonal array design is selected as experimental plan in Taguchi method. Basically, orthogonal array design is a general type of fractional factorial design and offers various combinations of control factors and their levels for each experiment. It can efficiently and effectively explore the whole factor space to simultaneously characterize the effects of multiple control factors. Based on the number of control factors and their level, one can determine an adequate orthogonal array design with specific experimental trials. Essentially, each row of an orthogonal array represents an experimental trial, and each column exhibits orthogonality property. The average output value of the selected objective at each factor level is then summarized in a graph or table format, respectively, termed response graph or response table. The response graph and table are mainly used for identifying important control factors and the associated optimal level that can achieve the optimal objective value. Furthermore, a statistical test, that is, analysis of variance (ANOVA), may sometime be applied to compare two or more means by way of assessing their variances. Typically, a significant variance implies a significant finding from the investigation. At last, the effectiveness of the optimal factor setting is confirmed through a confirmatory numerical experiment using the 3D nonlinear FE modeling.

\section{Experimental Testing}

5.1. Nanoindentation Testing. The nanoindentation continuous stiffness measurement (CSM) technique [21] is applied to characterize the mechanical properties of the dielectric layer (i.e., an organic PR material), namely, Young's modulus and hardness. During nanoindentation loading, a force signal with a small oscillation and a specified high excitation frequency is applied to the test specimen. Accordingly, the contact stiffness of materials that is to be characterized can be assessed from the output displacement response at the excitation frequency, from which the reduced or Young's modulus of the dielectric layer can be then assessed. The technique implies a primary advantage, in which Young's modulus can be calculated as a function of indentation depth. Moreover, posttest imaging is conducted to determine whether the testing would be made at the desired region.

5.2. Uniaxial Tensile/Compressive Testing. Uniaxial tensile/ compressive testing is the most commonly applied experimental technique for assessing the elastic-plastic behavior and properties of materials. In this study, uniaxial tensile/compressive tests are carried out using an Instron 8848 microtester with a displacement controlled loading and with a crosshead speed of $25 \mu \mathrm{m} / \mathrm{min}$ for compressive test and $100 \mu \mathrm{m} / \mathrm{min}$ for tensile test to characterize Young's modulus of the PI and PET materials. The test specimens are prepared in a dog-bone shape to ensure that the fracture of the specimens during testing would not occur at the two ends. Besides, the OCA adhesive consists of a very soft and compliant material, and it is not possible or straightforward to conduct uniaxial tensile testing on the material. Thus, in the present investigation, uniaxial compressive tests are also applied to evaluate Young's modulus of the OCA adhesive. During the progress of the compression, the force-displacement relationship is recorded, which can be further transformed into a stress-strain relationship based on finite element modeling. By the slope of the stress-strain curve, effective uniaxial Young's modulus of the specimens that are to be measured can be calculated.

5.3. Two-Point Bending Fatigue Test. Two-point bending fatigue test via a dynamic tester shown in Figure 5 is applied to characterize the bending fatigue behavior of the TSP structure designs. In the two-point bending test, a desired deformation or bending curvature is achieved through displacement control, and a cyclic load through displacement control is carried out at frequency of $1 \mathrm{~Hz}$. To facilitate the electrical resistance measurement, the bending fatigue 


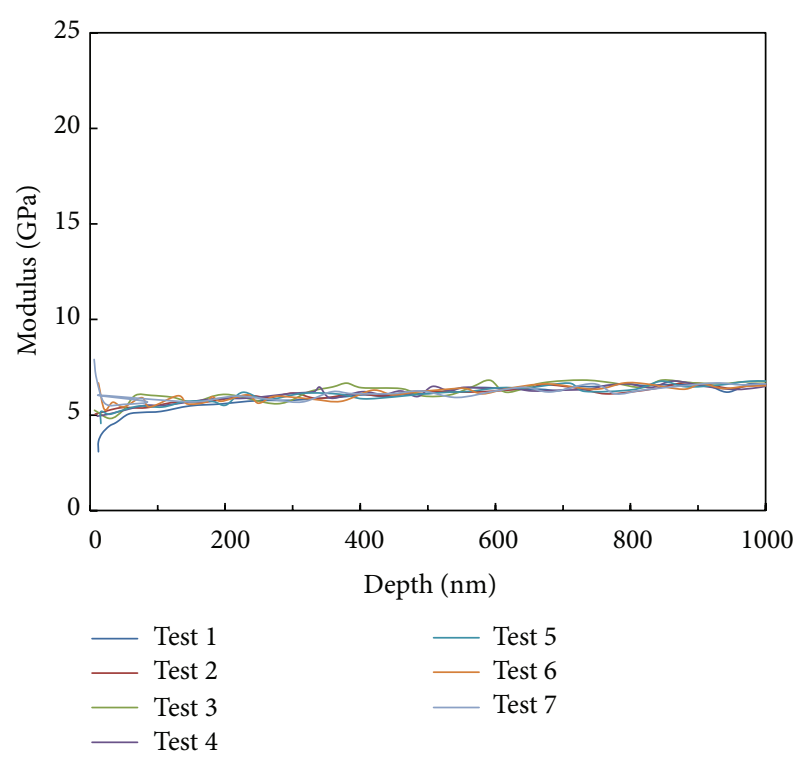

FIGURE 6: Reduced modulus of the organic PR material versus indentation depth.

test is incorporated with a two-point daisy chain resistance measurement. The bending fatigue failure is defined as a $10 \%$ increase of the electrical resistance.

\section{Results and Discussion}

6.1. Measured Young's Modulus of the Components. Young's modulus of the dielectric layer along the depth direction is first predicted through the nanoindentation CSM technique. To minimize measurement uncertainty, 7 nanoindentation experiments at randomly selected locations of the specimen are carried out. The arithmetic mean value of the 7 measurement results together with the arithmetic standard deviation is given. The curves of the reduced modulus $E_{r}$ versus the indentation depth are depicted in Figure 6. Note that the arithmetic mean value of the reduced modulus is obtained over the indentation depths ranging from 100 to $1000 \mathrm{~nm}$. It is found that the arithmetic mean reduced modulus of the dielectric layer together with the arithmetic standard deviation is about $6.15 \pm 0.40 \mathrm{GPa}$. The small standard deviation implies that the data are close to the mean and the consistency of the measurement results. To determine the corresponding Young's modulus, the effects of the indenter stiffness on measurements must be eliminated. Based on the following equation, $1 / E_{r}=\left(1-v_{s}^{2}\right) / E_{s}+\left(1-v_{i}^{2}\right) / E_{i}$, where $E_{s}\left(v_{s}\right)$ and $E_{i}\left(v_{i}\right)$ are Young's modulus (Poisson's ratio) of the specimen and indenter, respectively, together with the predicted reduced modulus, Young's modulus of the dielectric layer can be obtained. The arithmetic mean Young's modulus of the PR material as well as the arithmetic standard deviation is found to be about $5.29 \pm 0.78 \mathrm{GPa}$.

Young's moduli of the OCA adhesive of $20 \mu \mathrm{m}$ thickness and the PI and PET materials of 15 and $100 \mu \mathrm{m}$ thickness, respectively, are assessed through uniaxial compressive and tensile experiments. Likewise, four tests are attempted for

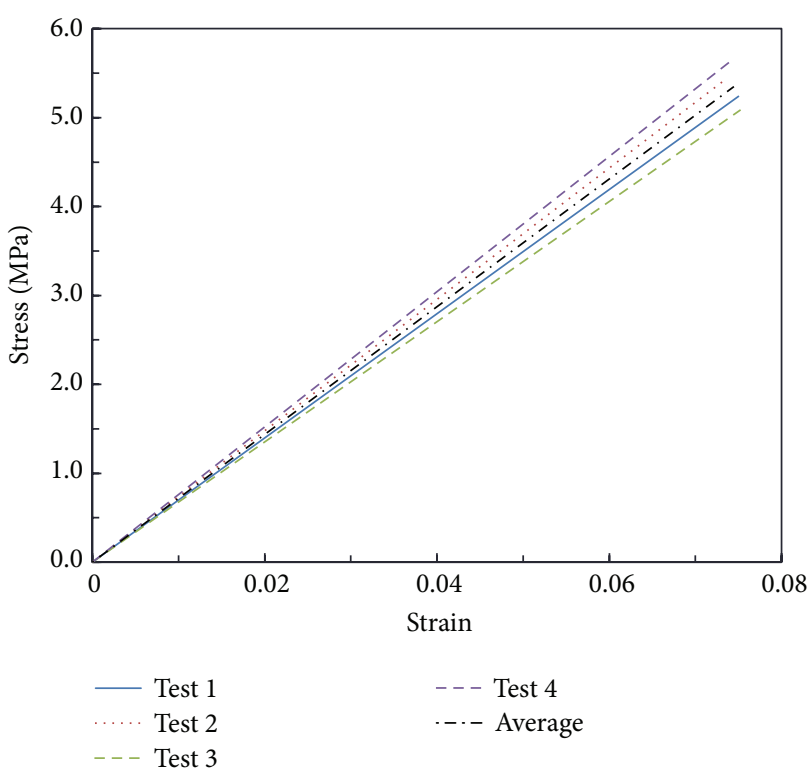

FIgURE 7: Compressive stress-strain curve of the OCA adhesive.

TABLE 1: Elastic properties of the components in the TDP structure.

\begin{tabular}{lcc}
\hline Material & Young's modulus $(\mathrm{GPa})$ & Poisson's ratio \\
\hline ITO & 106.5 & 0.475 \\
PR & 5.29 & 0.35 \\
PI & 2.7 & 0.34 \\
OCA & 0.072 & 0.35 \\
PET & 4.8 & 0.3 \\
PL & 0.07 & 0.35 \\
\hline
\end{tabular}

each of the specimens in order to lessen measurement uncertainty. The stress-strain relationships associated with these specimens are shown in Figures 7 and 8 . It is found that the average Young's modulus together with the arithmetic standard deviation associated with the OCA, PI, and PET materials is about $72.0 \pm 3.31 \mathrm{MPa}, 2.7 \pm 0.05 \mathrm{GPa}$, and $4.8 \pm 0.06 \mathrm{GPa}$. Clearly, the OCA adhesive holds a very low modulus. In addition, there is a small standard deviation, indicating a very good accuracy of the measurements. Table 1 lists the material properties used in the FE analysis, where all the materials are assumed to be linearly elastic.

6.2. Bending Behavior of the TDP Structure Designs. Through the proposed $3 \mathrm{D}$ nonlinear $\mathrm{FE}$ analysis, the $x$-dir. normal stresses and von Mises stresses of the ITO/PR, PI, and OCA components in the TSP structure under four-point bending with a radius of curvature of $0.75 \mathrm{~cm}$ are computed and illustrated in Figure 9, associated with the NPS, SPS, and FPS design. It is found that the bending load creates a significant bending stress (i.e., $x$-dir. normal stress and von Mises stress) in the ITO/PR conductive layer, either compressive or tensile, and the stress would increase with bending curvature. This can be attributed to the fact that not only does the layer have a high modulus, but also it consists of the largest distance from the neutral axis. As a result of the high tensile normal stress, 


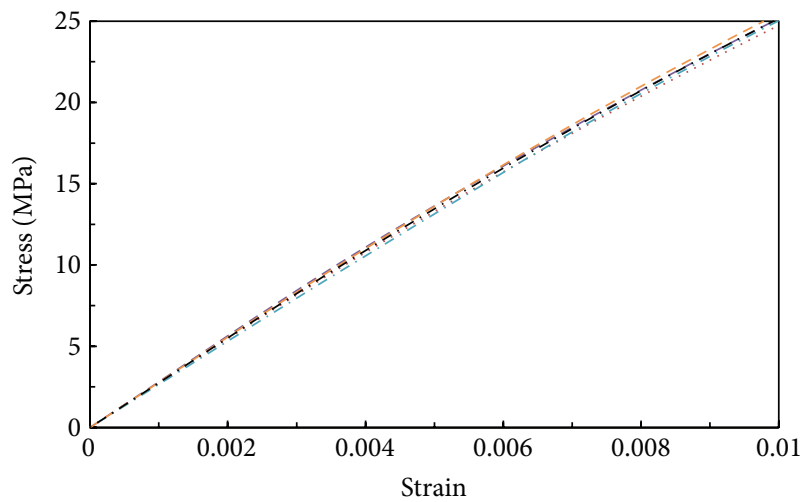

(a) PI

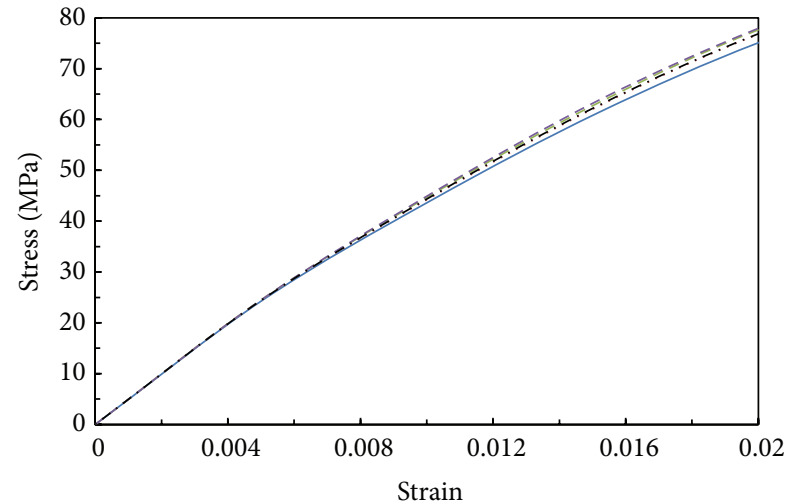

(b) PET

FIgURE 8: Tensile stress-strain curve for the PI and PET.

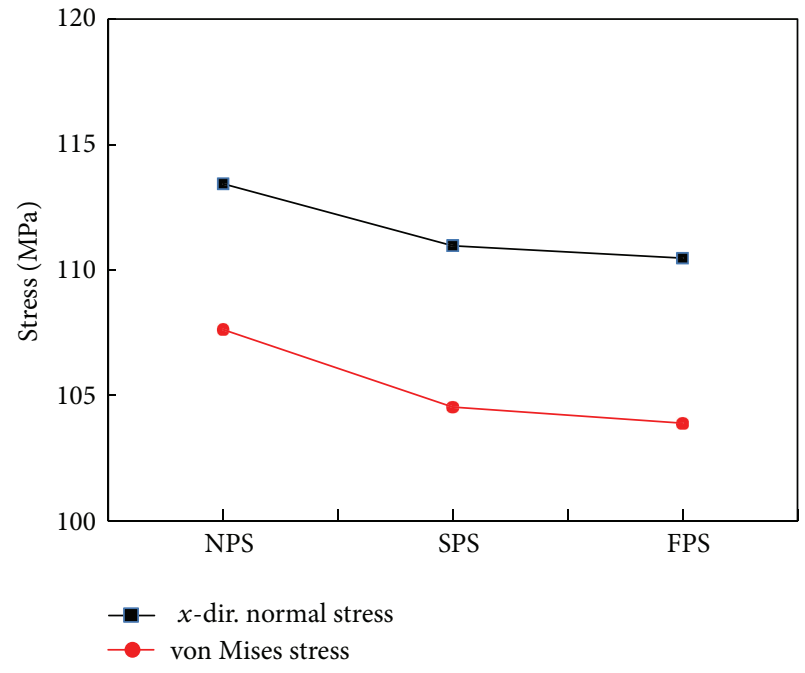

(a) ITO/PR conductive layer

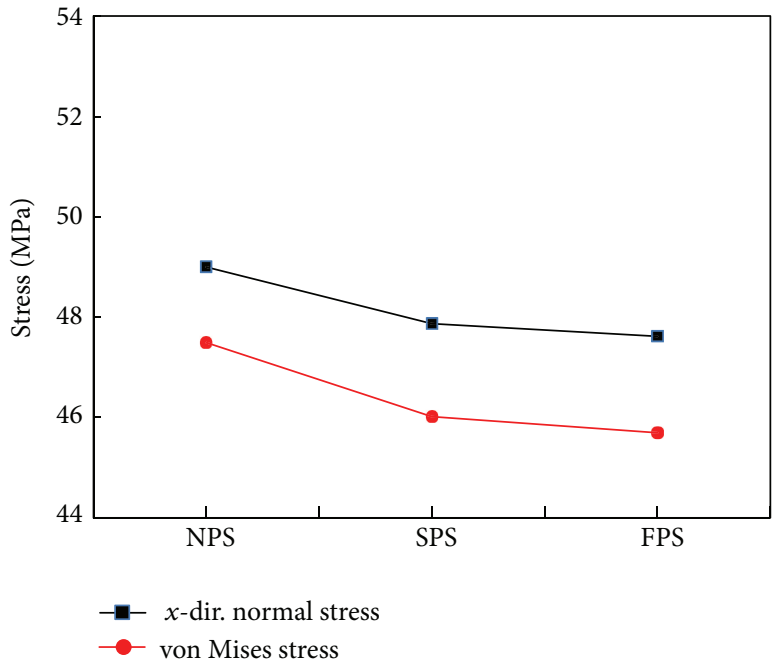

(b) PI layer

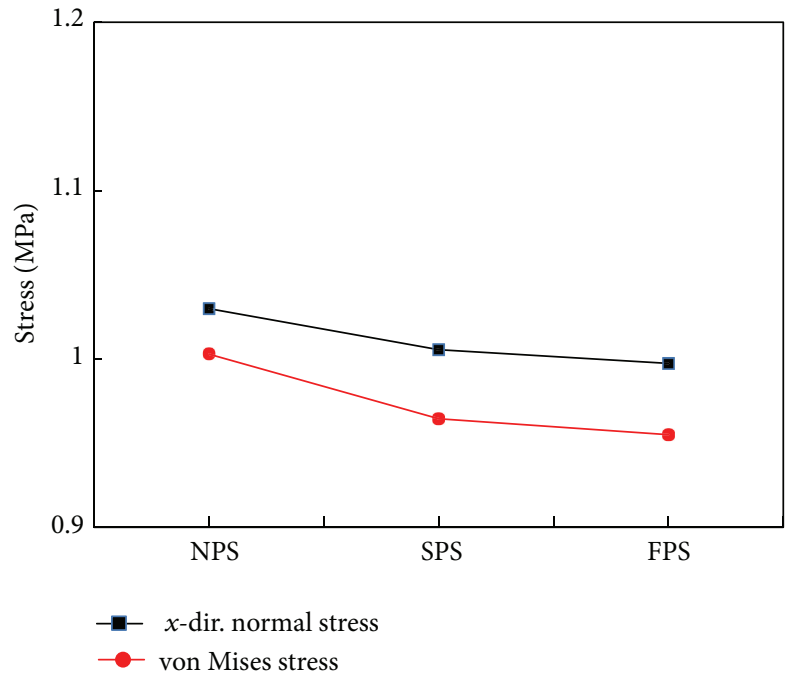

(c) OCA adhesive

FIGURE 9: Bending stresses at the ITO/PR, PI, and OCA components associated with the three protection structure designs. 
thin film cracking $[7,8]$ and even interfacial delamination, as shown in Figure 1(a), are two of the most commonly observed failure mechanisms in a laminate having a thin rigid film (e.g., ITO/PR) stacked on a thick compliant substrate (e.g., PET). On the other hand, while being subjected to an intense compressive stress, the laminate would suffer from local buckling at the interface of the thin rigid film and thick compliant substrate, leading to the well-known buckling delamination [7, 9-13], as shown in Figure 1(b). These failures are the major consideration or concern in the design of the ITO/PET laminate structure. As demonstrated in Figure 9, the issue is greatly improved by the use of the protection structure. The results show that the TDP structure with a protection structure would reduce both the $x$-dir. normal and von Mises stresses of these three components, that is, ITO/PR, PI, and OCA, compared to that without a protection structure, suggesting that the protection structures can be an effective means of enhancing the bending performance and even fatigue life of the touch panel laminate. In addition, it can be also observed that the SPS design would result in a slightly larger component stress than the FPS. Though the FPS would have a higher wear and water resistance and better protection of the touch panel laminate against bending failure than the former, the SPS design would have a better optical transparency and lower profile.

6.3. Parametric Analysis. The dependence of the bending stress in the ITO/PR conductive layer (i.e., $x$-dir. normal stress) on the material and geometry parameters of the components, that is, Young's modulus and thickness, is explored through parametric FE analysis. According to the preceding results, where the protection structure design turns out to be effective for enhancing the bending performance, thus, only the SPS and FPS type TDP structures are under investigation. Similarly, the TDP structures are subjected to a four-point bending load with a radius of curvature of $0.75 \mathrm{~cm}$. The results of the parametric analysis associated with the essential design factors are illustrated as follows.

(A) Effects of Young's Modulus of the Component. The substrate of the touch and display panels can be made of polymer materials, like polyethylene naphthalate (PEN), polymethyl methacrylate (PMMA), PET, polycarbonate (PC), polystyrene (PS), polyethersulfone (PES), polyimide (PI), polyvinyl alcohol (PVA), cyclic olefin polymer (COP), triacetylcellulose (TAC), biaxially oriented polystyrene (BOPS), and so forth and even glass. The influences of the elastic properties, in particular Young's modulus, of the components, namely, transparent touch panel substrate (i.e., PI in the original design), OCA, display panel substrate (i.e., PET in the original design), and the ratio of Young's modulus of the protection structure $\left(E_{\mathrm{PL}}\right)$ to that of the OCA adhesive $\left(E_{\mathrm{OCA}}\right)$, that is, $E_{\mathrm{PL}} / E_{\mathrm{OCA}}$, are examined on the bending stress (i.e., more specifically the $x$-dir. normal stress) of the ITO/PR conductive/dielectric layer, touch panel substrate, and OCA. In the parametric analysis, three different Young's moduli are considered for each component, in which they are 70, 90 , and $110(\mathrm{MPa})$ for the OCA, 3.6, 4.8, and $6.0(\mathrm{GPa})$ for the display panel substrate, 2.0, 2.7, and $3.4(\mathrm{GPa})$ for the touch panel substrate, and 1.0, 1.5, and 2.0 for the $E_{\mathrm{PL}} / E_{\mathrm{OCA}}$ ratio. First of all, the effects of the OCA Young's modulus are displayed in Figure 10. The results reveal that an increasing OCA Young's modulus tends to decrease the normal stress of the conductive/dielectric layer and PI substrate but enlarge that of the OCA. In addition, the increase of the $E_{\mathrm{PL}} / E_{\mathrm{OCA}}$ ratio would reduce the normal stress of these components. Next, the influences of Young's modulus of the display panel substrate are given in Figure 11. The figure demonstrates that the normal stress of these components would increase with Young's modulus of the display panel substrate. The result agrees well with the experimental finding of Lim et al. [10], where they found that a polymer substrate with a lower Young's modulus would hold better mechanical and electrical stability against bending stresses. Moreover, the effects of Young's modulus of the touch panel substrate are presented in Figure 12. It is clear to see that the increase of Young's modulus of the touch panel substrate would reduce the normal stress of the ITO/PR conductive/dielectric and OCA components but raise that of the touch panel substrate. From these parametric analysis results, it is, again, found that the FPS design (the solid line) outperforms the SPS (the dotted line) in terms of reduced normal stress.

(B) Effects of the Geometry of the Components. We first examine the effects of the thickness of the components on the bending stress of the TDP structure, and the results are shown in Figure 13. Note that only the SPS type TDP structure is considered in the investigation since, according to the preceding results, there is a comparable result trend for both the SPS and FPS designs. In the parametric analysis, the thickness of the components varies from $75 \%$ to $125 \%$ of their original value as described in Section 2. However, that of the SPS (i.e., " $T$ " in Figure 2(b)) changes from 0 to $50 \%$. The figure exhibits that, among the thicknesses of the components under investigation, the thickness of the SPS and PET substrate would have a greater impact on the $x$-dir. normal stress of the components. In addition, the decrease of the thickness of the OCA and the PET and PI substrate and the increase of the SPS thickness would lower the normal stress of the ITO/PR layer and PI substrate. In addition, the lessening of the thickness of the PET substrate and OCA and the increase of the thickness of the SPS and PI substrate would diminish the normal stress of the OCA layer. The results imply that a more compliant substrate tends to yield a less normal stress in the ITO/PR layer, thereby leading to better bending performance. The result is also consistent with the experimental finding of Lim et al. [10]. By contrast, the thickness effect of the ITO/PR layer on the $x$-dir. normal stress of the components is much less pronounced. In addition to the thickness of the SPS, the dependence of the SPS width (i.e., " $W_{1}$ " in Figure 2(b)) on the bending stress is also examined. Three different SPS widths are considered, namely, $0.4,0.8$, and $1.2 \mathrm{~mm}$, and the analysis results are shown in Figure 14. It turns out that the bending stress of these components is dependent on the SPS width, where an increasing SPS width would, likewise, lead to a smaller bending stress, thereby resulting in a better bending performance. 


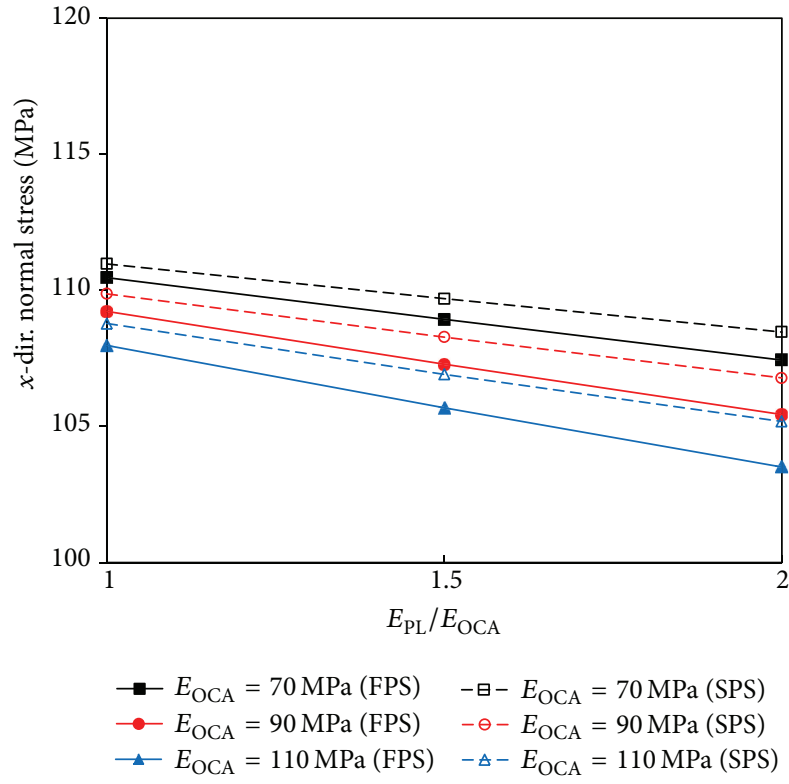

(a) ITO/PR layer

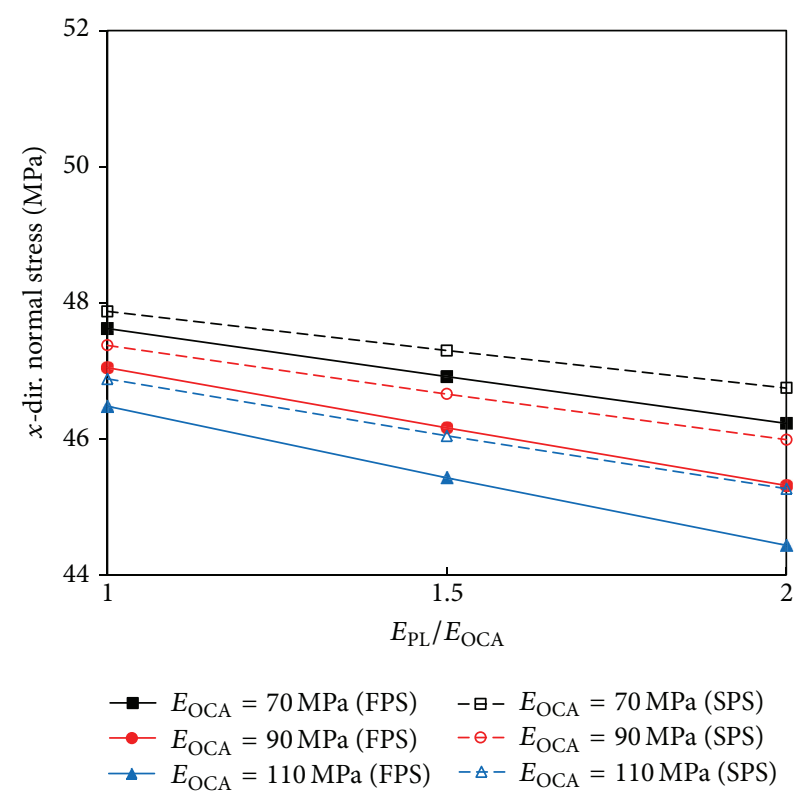

(b) PI substrate

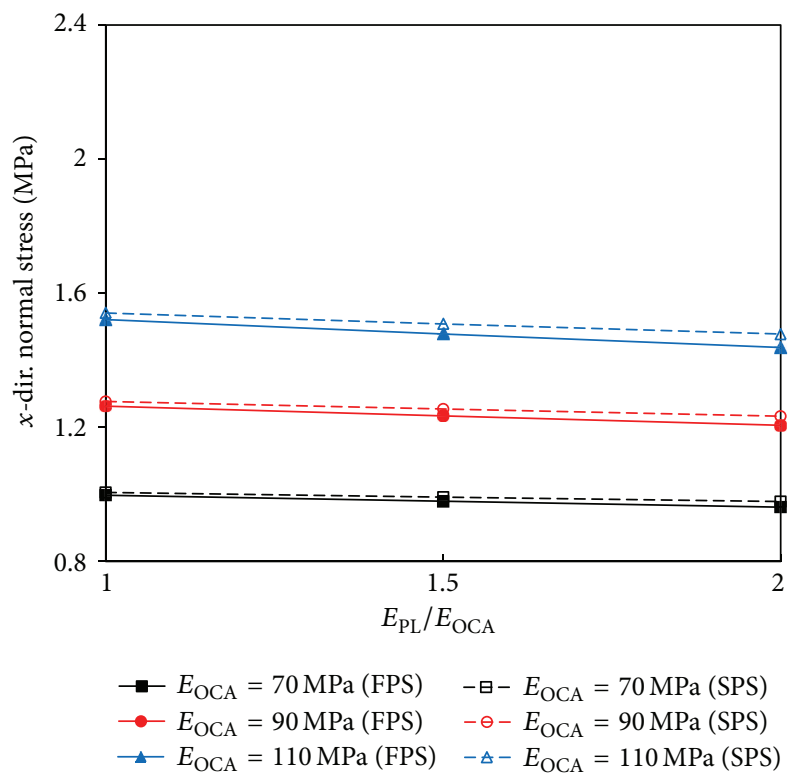

(c) OCA

FIGURE 10: Effects of OCA Young's modulus and $E_{\mathrm{PL}} / E_{\mathrm{OCA}}$ ratio on the bending stress of the components.

6.4. Experimental Bending Fatigue Test Results. According to the preceding parametric analysis, an increasing SPS thickness and width would help reduce the component stress, potentially leading to a better bending fatigue life. To validate the proposed FE modeling, a two-point bending fatigue test in conjunction with a two-point daisy chain resistance measurement is carried out on the TDP structure with an SPS. In the investigation, three different SPS thicknesses (i.e., " $W_{2}$ " in Figure 2(b)), namely, 25, 50, and $100(\mu \mathrm{m})$, and SPS widths $\left(W_{1}\right)$, that is, $0.3,0.6$, and $1.2(\mathrm{~cm})$, are considered. The specimens are subjected to cyclic bending with a radius of curvature of $0.75 \mathrm{~cm}$. The test results are shown in Figure 15.
It shows that an increasing SPS thickness and width can all improve the bending fatigue life of the TDP structure. The results agree well with those of the FE modeling. The TDP structure under bending tends to generate corrugation. The corrugation lengths after a certain number of bending cycles associated with the $0 \mathrm{~cm}, 0.3 \mathrm{~cm}, 0.6 \mathrm{~cm}$, and $1.2 \mathrm{~cm}$ wide SPS are listed in Table 2. An example of the corrugation failure associated with two different SPS designs, that is, $W_{1}=0 \mathrm{~cm}$ and $1.2 \mathrm{~cm}$, after 1000 bending cycles is shown in Figure 15(b). It is found that there is no corrugation failure on the side with a $1.2 \mathrm{~cm}$ wide SPS but an excessive corrugation on the side without an SPS. The table shows 


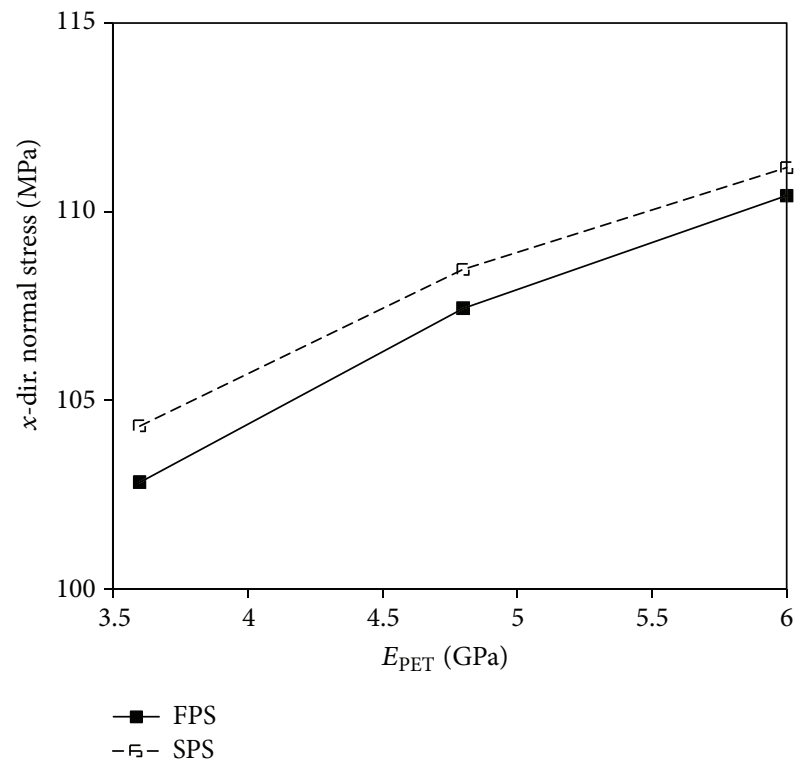

(a) ITO/PR layer

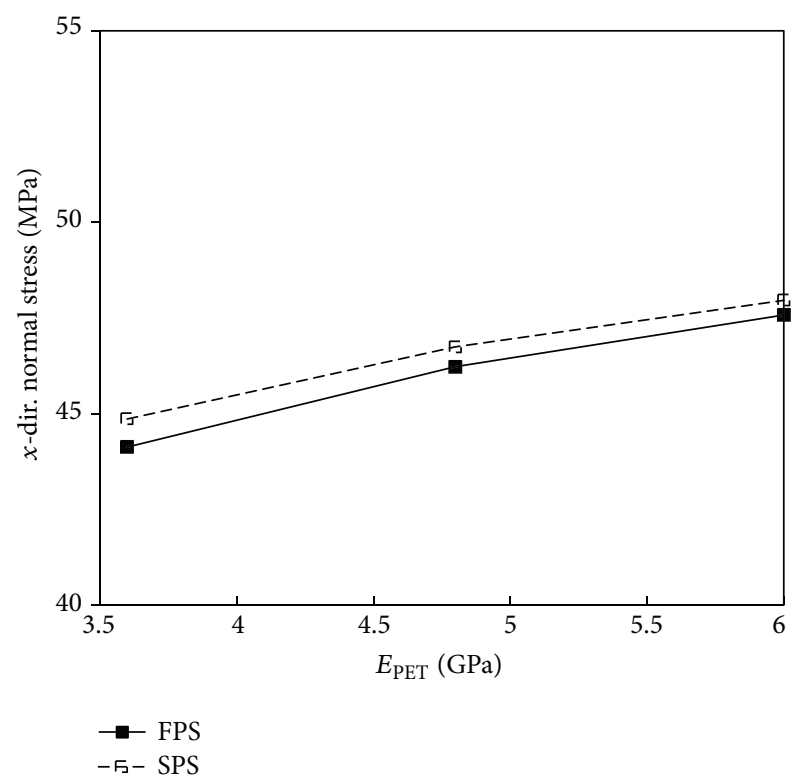

(b) PI substrate

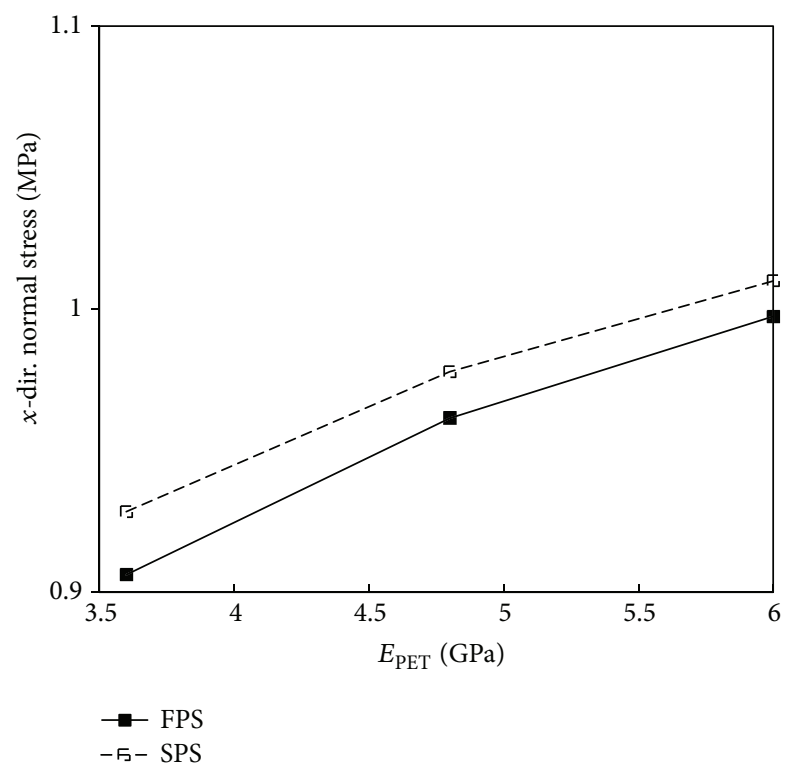

(c) OCA

FIGURE 11: Effects of Young's modulus of display panel substrate on the bending stress of the components.

that corrugation failure would not occur even after 10000 bending cycles for the $0.6 \mathrm{~cm}$ and $1.2 \mathrm{~cm}$ wide SPS. Though at only 1000 bending cycles the $0.3 \mathrm{~cm}$ wide SPS would yield a $2 \mathrm{~cm}$ long corrugation, it is still much superior to the original design (i.e., TDP without the protection structure design), in which there is a $9.5 \mathrm{~cm}$ long corrugation at the same bending cycle number. Besides, it is also clear to see that the corrugation failure on the side of the TDP specimen without the protection structure tends to initiate at the side edge and further propagates inward toward the center, suggesting that there is a stress concentration at the side edge region due to geometry and material discontinuity. The improvement in the bending fatigue performance of the TDP with the protection structure design could be mainly due to not only the reduction of the bending stress (see Figure 9) as a result of (1) the increase of the bending rigidity, (2) the elevation of the neutral axis of the TDP's cross section to a position closer to the ITO/PR layer, and (3) share of the bending stress by the side protection structure but also the increase of the bond line length. The analysis results also reveal that the larger the SPS width and thickness, the less the corrugation length under the same bending cycles and the better the bending fatigue life.

6.5. Taguchi Experimental Design. To further upgrade the bending performance of the TDP structure consisting of an 


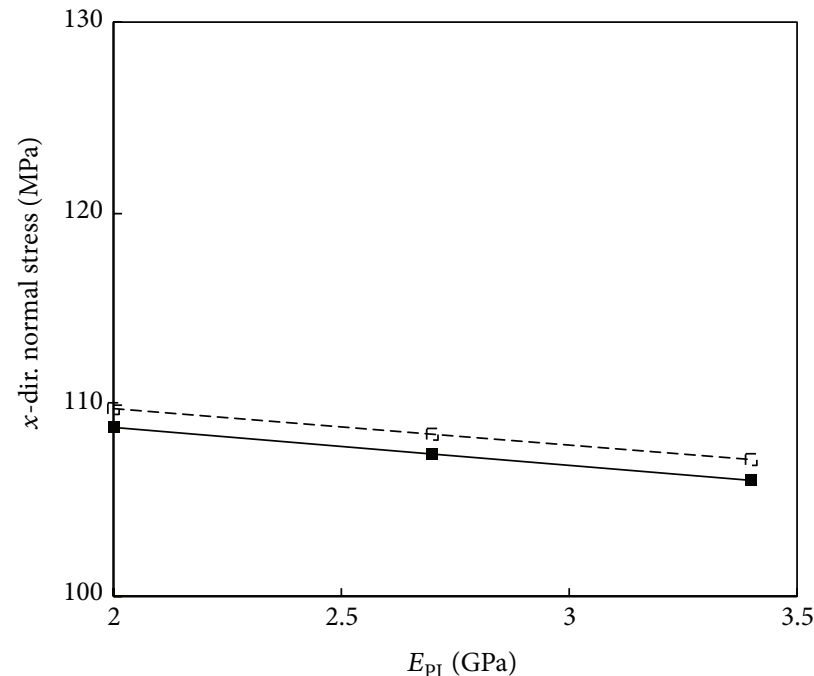

$\rightarrow-$ FPS

$-5-$ SPS

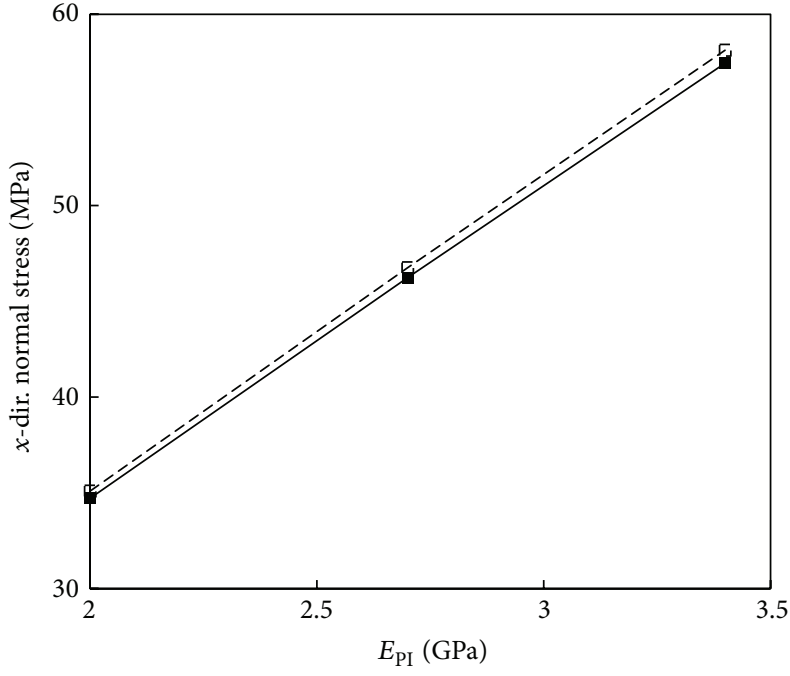

$\rightarrow$ FPS

$-5-$ SPS

(a) ITO/PR layer

(b) Touch panel substrate

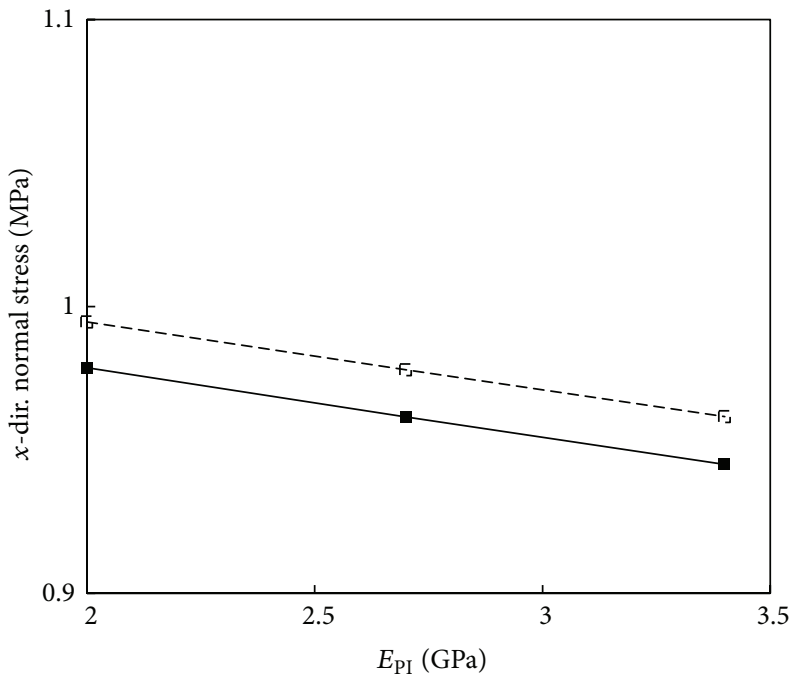

- FPS

- - - SPS

(c) OCA adhesive

FIGURE 12: Effects of Young's modulus of touch panel substrate on the bending stress of the components.

TABLE 2: Corrugation failure length versus cycle times associated with four different SPS widths.

\begin{tabular}{lc}
\hline$W_{1}(\mathrm{~cm})$ & Corrugation length $(\mathrm{cm}) /($ Cycle times $)$ \\
\hline 1.2 & $0 /(>10000)$ \\
0.6 & $0 /(>10000)$ \\
0.3 & $2 /(1000)$ \\
0 & $9.5 /(1000)$ \\
\hline
\end{tabular}

SPS, Taguchi experimental design is performed. Six key control factors are selected for the experimental design, in which they are the thickness of the display panel substrate (A) and SPS (B) and Young's modulus of the display panel substrate (C), OCA adhesive (D), touch panel substrate (E), and SPS (F). Three levels for each control factor are selected in order to account for the nonlinear effect. Table 3 presents the selected control factors and levels. In the table, the starting level of the control factors is identified by a bold style. Nominal $\pm 25 \%$ variations of the value of Level 2 are used to define the upper and lower levels. The focus (selected objective) of the design is to minimize the $x$-dir. normal stress of the ITO/PR layer. The experimental design applies the validated 3D nonlinear FE model for bending stress assessment, and an $L_{18}\left(2^{1} \times 3^{7}\right)$ 

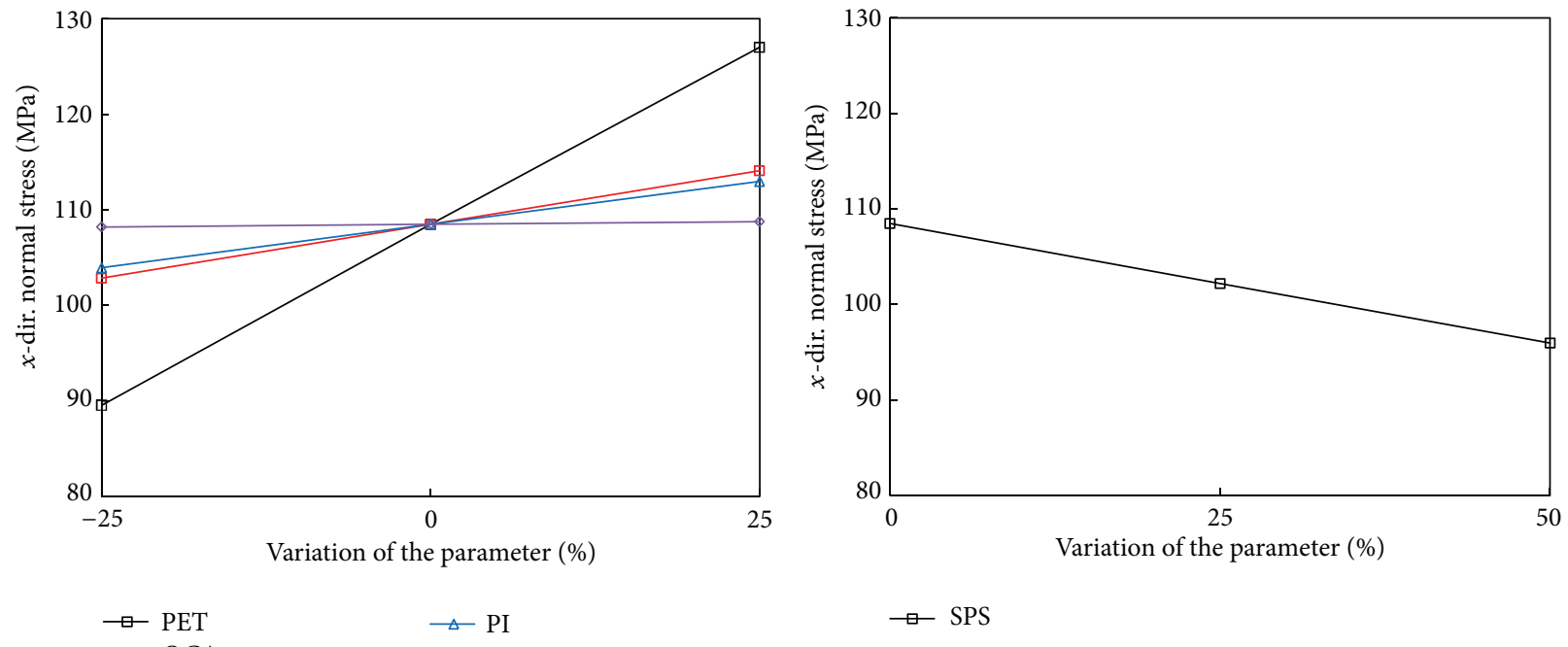

(a) ITO/PR layer
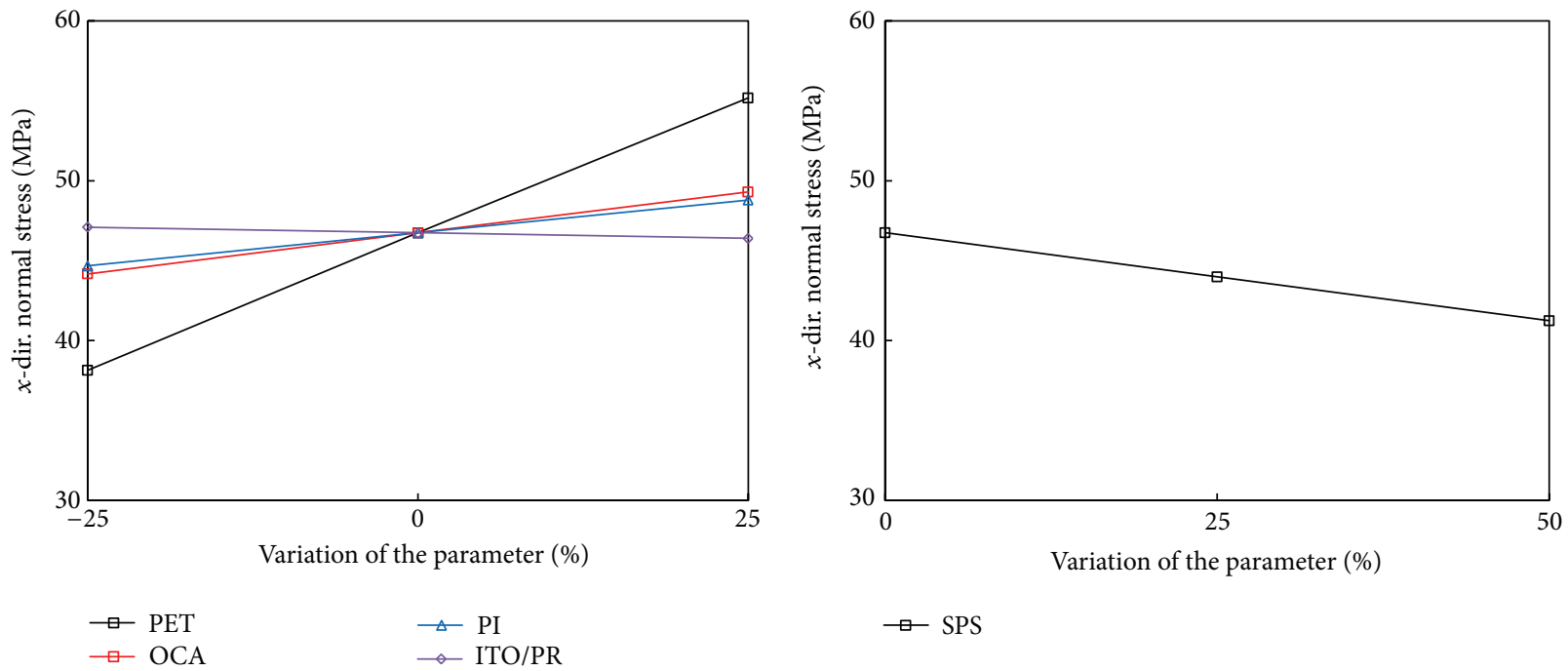

$\rightarrow$ SPS

(b) PI substrate
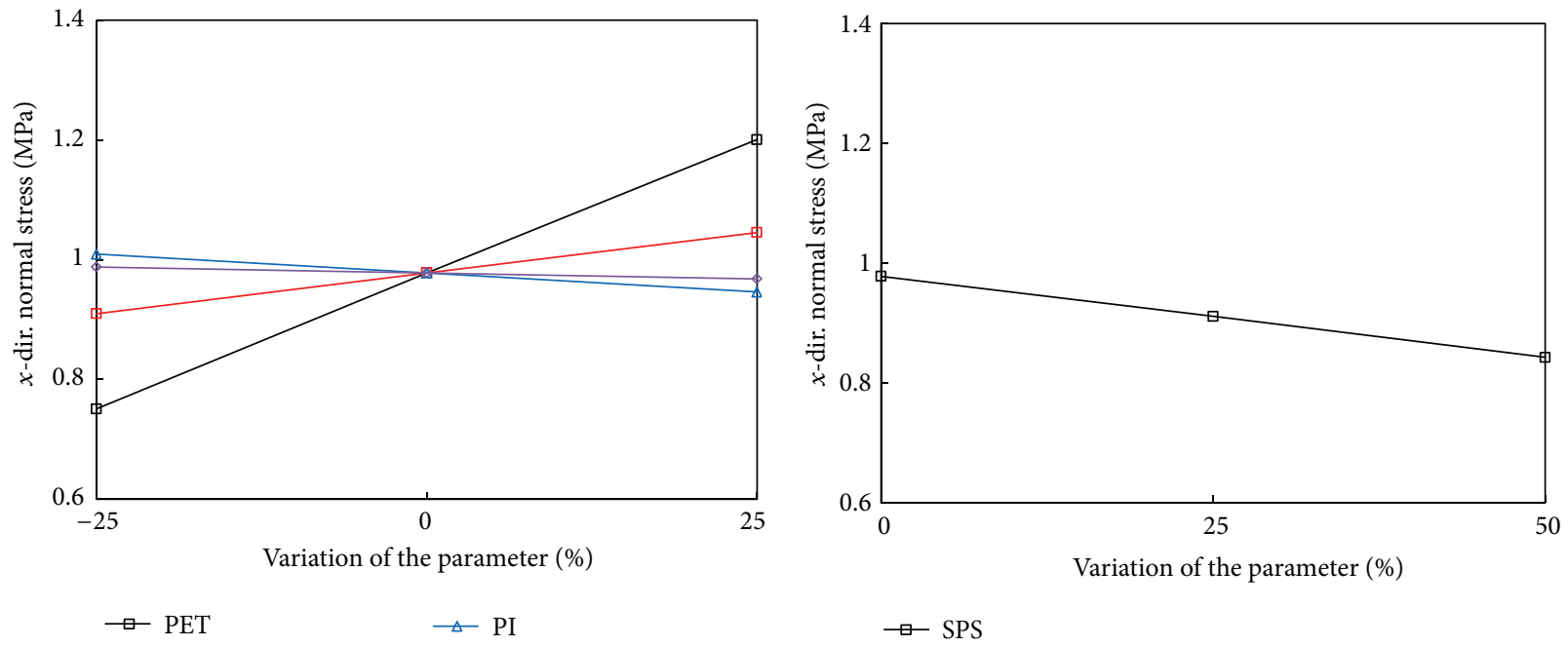

$\begin{array}{ll}\rightarrow \text { PET } & \rightarrow \text { PI } \\ \rightarrow \text { OCA } & \rightarrow \text { ITO/PR }\end{array}$

(c) OCA adhesive

FIGURE 13: Effects of the thickness of the components on the bending stress. 


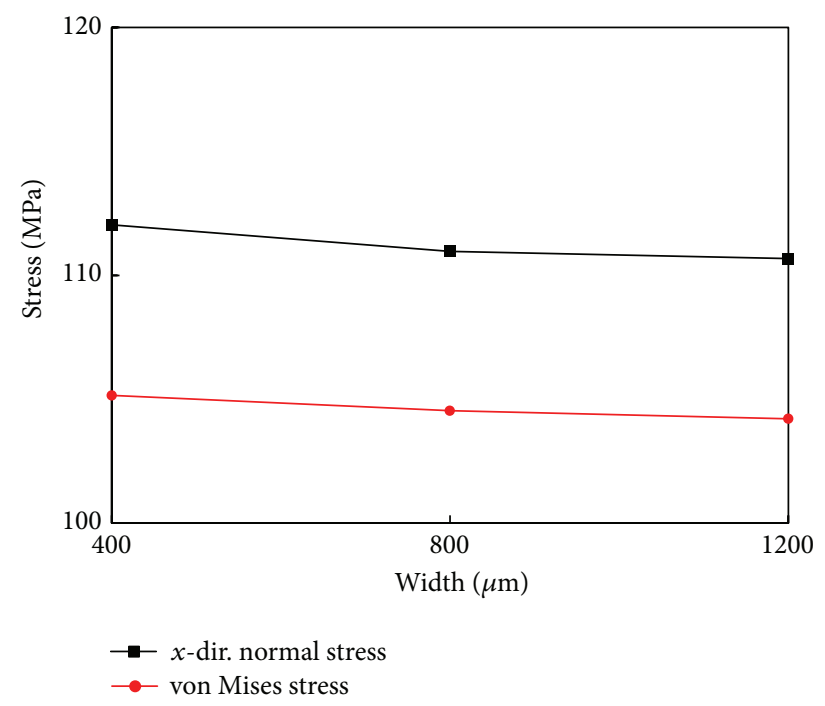

(a) ITO/PR layer

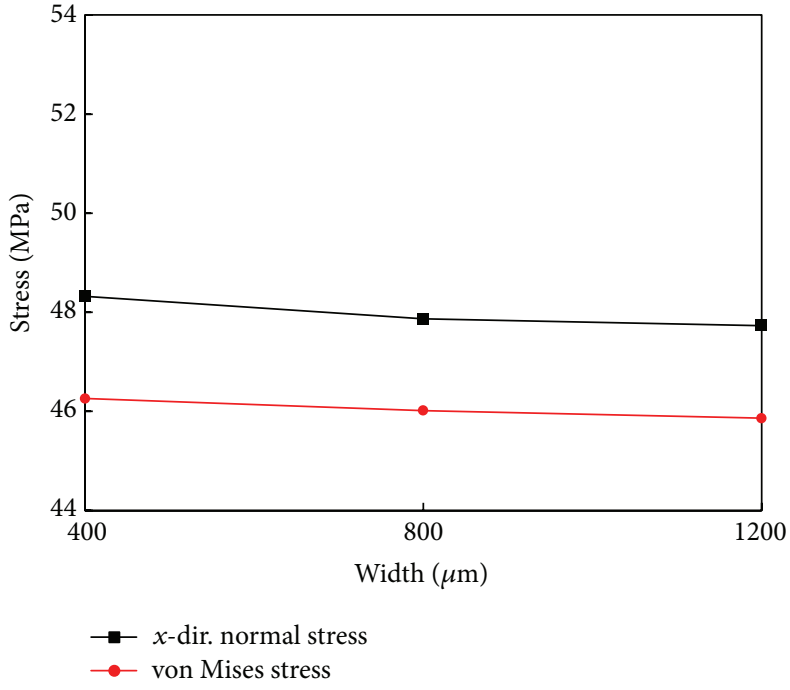

(b) PI substrate

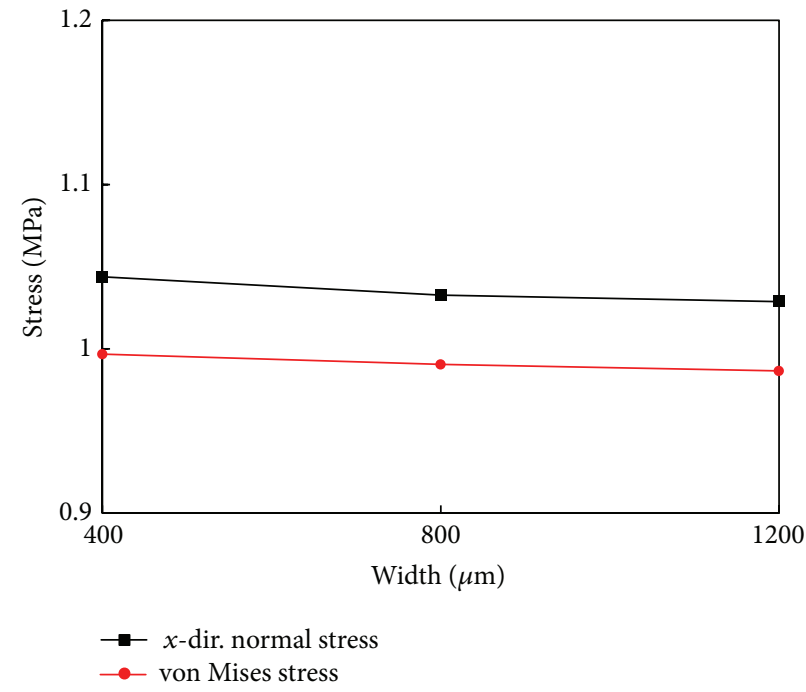

(c) OCA adhesive

FIGURE 14: Effects of the SPS width on the bending stress.

TABLE 3: Design parameters and their levels.

\begin{tabular}{|c|c|c|c|c|}
\hline & Control factors & Level 1 & Level 2 & Level 3 \\
\hline A & $\begin{array}{l}\text { Thickness of display panel } \\
\text { substrate }(\mu \mathrm{m})\end{array}$ & 75 & 100 & 125 \\
\hline B & Thickness of SPS $(\mu \mathrm{m})$ & 50 & 66.6 & 83.2 \\
\hline $\mathrm{C}$ & $\begin{array}{l}\text { Young's modulus of display } \\
\text { panel substrate }(\mathrm{MPa})\end{array}$ & 3600 & 4800 & 6000 \\
\hline $\mathrm{D}$ & $\begin{array}{l}\text { Young's modulus of OCA } \\
(\mathrm{MPa})\end{array}$ & 70 & 93.3 & 116.6 \\
\hline $\mathrm{E}$ & $\begin{array}{l}\text { Young's modulus of touch } \\
\text { panel substrate }(\mathrm{MPa})\end{array}$ & 2000 & 2700 & 3370 \\
\hline $\mathrm{F}$ & $\begin{array}{l}\text { Young's modulus of SPS } \\
(\mathrm{MPa})\end{array}$ & 105 & 140 & 175 \\
\hline
\end{tabular}

orthogonal array is adopted, as shown in Table 4, implying that there are two dummy control factors, thus leading to two dummy columns. For each experimental trial, corresponding to a row vector of the orthogonal array, the calculated $x$-dir. normal stress of the ITO/PR layer is also shown in Table 4. The average normal stresses of each control factor at different levels and also the associated variances are calculated and summarized in the response graph in Figure 16, where "A1," for example, denotes the control factor "A" at Level 1. It is evident to see that the thickness of the display panel substrate presents the greatest influence on the bending stress of the ITO/PR layer, followed by the thickness of the SPS and Young's modulus of the display panel substrate. The set of the minimal value of each curve, representing 


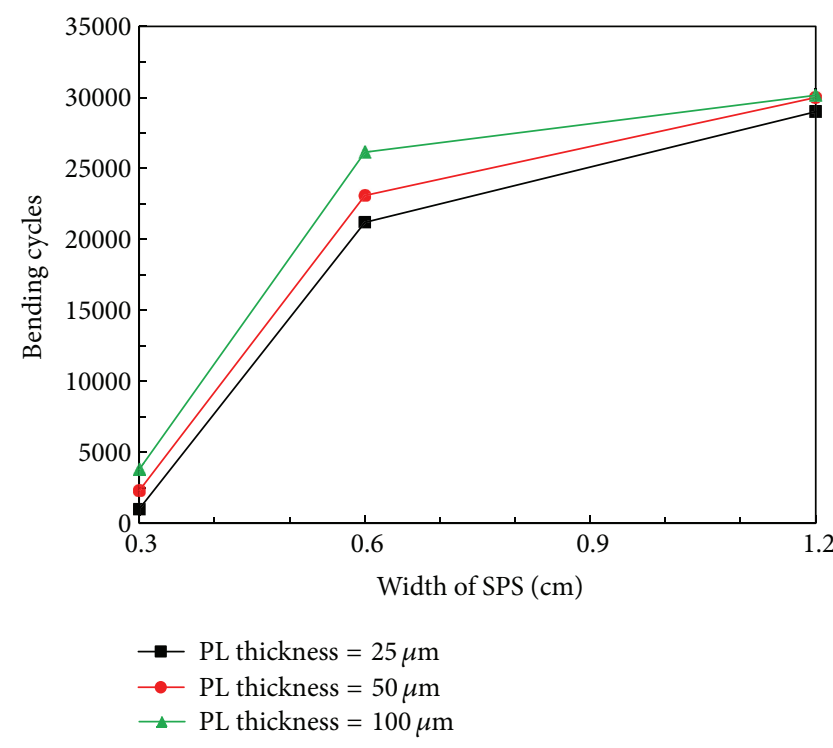

(a) Bending fatigue life versus SPS width

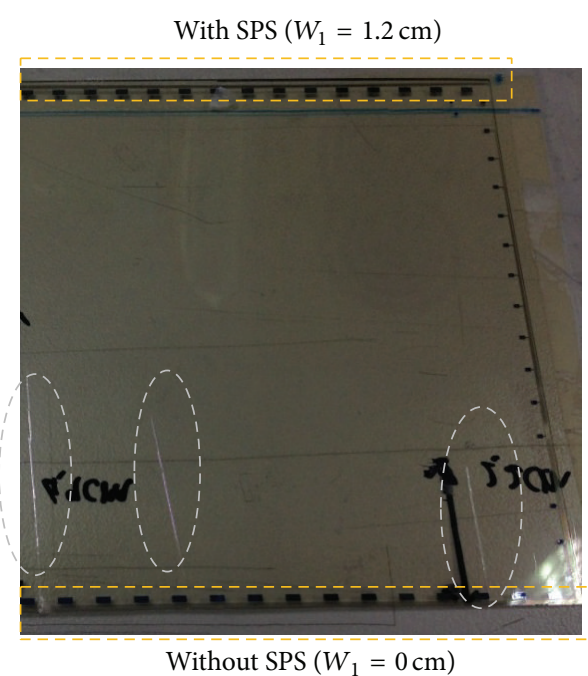

(b) Corrugation failure versus SPS width

FIGURE 15: Effects of SPS thickness and width on bending performance of the TDP structure.

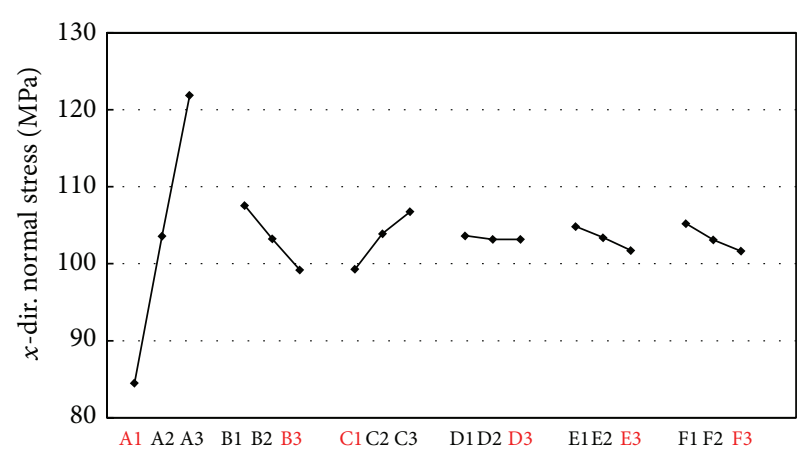

FIGURE 16: Effect of control factors on the bending stress of the ITO/ PR conductive layer.

the best thermal performance, is considered as the optimum design as it yields the minimal bending stress. The optimal level set of these control factors is A1, B3, C1, D3, E3, and F3, corresponding to a $75 \mu \mathrm{m}$ thick display panel substrate, a $83.2 \mu \mathrm{m}$ thick SPS, a display panel substrate with a Young's modulus of $3600 \mathrm{MPa}$, an OCA with a Young's modulus of 116.6 MPa, a touch panel substrate with a Young's modulus of $3370 \mathrm{MPa}$, and an SPS with a Young's modulus of $175 \mathrm{MPa}$. FE modeling of the optimal design is further conducted, and the result is shown in Table 5. The bending stress of the original TDP design (NPS) is also shown in the table. The table demonstrates that the Taguchi experimental design can not only dramatically reduce the bending stress of the ITO/PR layer from 113.4 MPa to 71.1 MPa, but also considerably lessen that of the touch panel substrate and OCA.
TABLE 4: $L_{18}\left(2^{1} \times 3^{7}\right)$ orthogonal array and the calculated $x$-dir. normal stress of the ITO/PR layer.

\begin{tabular}{lccccccc}
\hline Exp. number & $\mathrm{A}$ & $\mathrm{B}$ & $\mathrm{C}$ & $\mathrm{D}$ & $\mathrm{E}$ & $\mathrm{F}$ & $\mathrm{ITO} / \mathrm{PR}$ \\
\hline 1 & 1 & 1 & 1 & 1 & 1 & 1 & 84.52 \\
2 & 1 & 2 & 2 & 2 & 2 & 2 & 103.59 \\
3 & 1 & 3 & 3 & 3 & 3 & 3 & 121.88 \\
4 & 2 & 1 & 1 & 2 & 2 & 3 & 107.55 \\
5 & 2 & 2 & 2 & 3 & 3 & 1 & 103.24 \\
6 & 2 & 3 & 3 & 1 & 1 & 2 & 99.19 \\
7 & 3 & 1 & 2 & 1 & 3 & 2 & 99.30 \\
8 & 3 & 2 & 3 & 2 & 1 & 3 & 103.91 \\
9 & 3 & 3 & 1 & 3 & 2 & 1 & 106.77 \\
10 & 1 & 1 & 3 & 3 & 2 & 2 & 103.64 \\
11 & 1 & 2 & 1 & 1 & 3 & 3 & 103.18 \\
12 & 1 & 3 & 2 & 2 & 1 & 1 & 103.17 \\
13 & 2 & 1 & 2 & 3 & 1 & 3 & 104.85 \\
14 & 2 & 2 & 3 & 1 & 2 & 1 & 103.40 \\
15 & 2 & 3 & 1 & 2 & 3 & 2 & 101.73 \\
16 & 3 & 1 & 3 & 2 & 3 & 1 & 105.23 \\
17 & 3 & 2 & 1 & 3 & 1 & 2 & 103.10 \\
18 & 3 & 3 & 2 & 1 & 2 & 3 & 101.65 \\
\hline
\end{tabular}

\section{Conclusions}

In the study, the polymer-based thin protection structure is proposed and demonstrated so as to protect the touch panel laminate against bending fracture and thus enhance its bending and reliability performance. Two different types of 
TABLE 5: Comparison of the original and optimal results.

\begin{tabular}{lcc}
\hline Components & NPS & Optimal design \\
\hline ITO/PR & 113.4 & 71.1 \\
Touch panel substrate & 49.0 & 37.3 \\
OCA & 1.03 & 0.89 \\
\hline
\end{tabular}

protection structure, namely, the FPS and SPS, are proposed. The mechanical behavior of the TDP laminate without or with the protection structures under four-point bending is examined using 3D geometry-nonlinear $\mathrm{FE}$ analysis and experimental fatigue testing, where the needed elastic properties of the components in the FE analysis are experimentally determined from nanoindentation and uniaxial tensile/compressive testing. The calculated results are compared with each other and with those of the original TDP without a protection structure. Finally, a design guideline and an optimal factor setting design for enhanced bending performance are sought through parametric $\mathrm{FE}$ analysis and Taguchi experimental design. Some essential concluding remarks are drawn as follows.

(1) Young's modulus of the organic PR dielectric material estimated from the nanoindentation test is about 5.3 GPa and that of the OCA, PI, and PET materials derived from the uniaxial compressive/tensile test is about $72.0 \mathrm{MPa}, 2.7 \mathrm{GPa}$, and $4.8 \mathrm{GPa}$. It is clear that the OCA has a very low modulus, and the measurement data has a very small standard deviation, indicating the consistency of the measurement results.

(2) The analysis results reveal that excessive bending would produce considerable tensile and compressive bending stresses on the ITO/PR conductive/dielectric layer, and the stress would increase with bending curvature. The high tensile and compressive bending stresses are the main cause of several well-reported failures of a laminate with a thin rigid film coating on a thick compliant substrate, such as thin film cracking and delamination, and thus become the major consideration or concern in the design of the laminate structure.

(3) Both the SPS and FPS designs are capable of protecting the touch panel laminate from bending fatigue, leading to a better bending performance, such as bendability and fatigue life. In addition, the FPS outperforms the SPS in bending stress, suggesting that the FPS design can have a better protection of the touch panel laminate against bending fracture. In spite of lower wear and water resistance and somewhat smaller protection capability, the SPS design may still be an ideal option because of its better optical transparency and lower profile.

(4) The parametric study and Taguchi experimental design show that the bending-induced normal stress of the components would be most affected by the thickness of the SPS and display panel substrate and Young' modulus of the display panel substrate. In addition, the decrease of the thickness of the display and touch panel substrates and OCA and the increase of the SPS thickness would reduce the bending stress of the ITO/PR layer and touch panel substrate. Besides, the decrease of the thickness of the display panel substrate and OCA and the increase of the thickness of the SPS and touch panel substrate would lessen the bending stress of the OCA. Similar to the SPS thickness, the width of the SPS would also play a considerable role in the bending stress of these components, in which it would increase with the SPS width, thus causing a worse bending performance.

(5) The simulation results are in good agreement with the present experimental bending fatigue test data and also the literature findings, demonstrating the effectiveness of the proposed 3D nonlinear FE modeling.

(6) The Taguchi experimental design shows that the optimal factor setting for the TDP structure with an SPS is A1, B3, C1, D3, E3, and F3, which turns out to not only radically lessen the bending stress of the ITO/PR layer but also noticeably reduce that of the touch panel substrate and OCA.

\section{Conflict of Interests}

The authors declare that there is no conflict of interests regarding the publication of this paper.

\section{Acknowledgments}

This work was supported by the Industrial Technology Research Institute, Taiwan, and National Science Council, Taiwan, under Contract nos. MOST 103-2221-E-035-024MY3 and NSC101-2221-E-007-009-MY3.

\section{References}

[1] J.-M. Park, J.-S. Hong, J.-Y. Yang et al., "Bending effects of indium-zinc oxide thin films deposited on polyethylene terephthalate substrate by radio frequency magnetron sputtering," Journal of the Korean Physical Society, vol. 48, no. 6, pp. 15301533, 2006.

[2] B. Sim, E.-H. Kim, J. Park, and M. Lee, "Highly enhanced mechanical stability of indium tin oxide film with a thin $\mathrm{Al}$ buffer layer deposited on plastic substrate," Surface \& Coatings Technology, vol. 204, no. 3, pp. 309-312, 2009.

[3] K. Alzoubi, M. M. Hamasha, S. Lu, and B. Sammakia, "Bending fatigue study of sputtered ITO on flexible substrate," Journal of Display Technology, vol. 7, no. 11, pp. 593-600, 2011.

[4] D. R. Caims, D. C. Paine, and G. P. Carwford, "The mechanical reliability of sputter-coated indium tin oxide polyester substrates for flexible display and touchscreen applications," $M R S$ Proceedings, vol. 666, 2001.

[5] J.-S. Oh, Y.-R. Cho, K.-E. Cheon, M. A. Karim, and S. J. Jung, "Failure mechanism of patterned ITO electrodes on flexible substrate under static and dynamic mechanical stresses," Solid State Phenomena, vol. 124-126, no. 1, pp. 411-414, 2007.

[6] Z. Yu, L. Xiang, W. Xue, and H. Wang, "The bending properties of flexible ITO films," in Asia Optical Fiber Communication and 
Optoelectronic Exposition and Conference, pp. 148-150, October 2007.

[7] P. C. P. Bouten, P. J. Slikkerveer, and Y. Leterrier, "Chapter 6: mechanics of ITO on plastic substrates for flexible," in Flexible Flat Panel Displays, G. P. Grawford, Ed., p. 117, John Wiley \& Sons, Chichester, UK, 2005.

[8] M.-K. Yeh, L.-Y. Chang, M.-R. Lu, H.-C. Cheng, and P.H. Wang, "Bending stress analysis of flexible touch panel," Microsystem Technologies, vol. 20, no. 8-9, pp. 1641-1646, 2014.

[9] G. A. Kardomateas, "Snap buckling of delaminated composites under pure bending," Composites Science and Technology, vol. 39, no. 1, pp. 63-74, 1990.

[10] C. Lim, J. Park, Y. Kim, and J. Han, "Mechanical and electrical stability indium-tin-oxide coated polymer substrates under continuous bending stress condition," Journal of International Council on Electrical Engineering, vol. 2, no. 3, pp. 237-241, 2014.

[11] B. Cotterell and Z. Chen, "Buckling and cracking of thin films on compliant substrates under compression," International Journal of Fracture, vol. 104, no. 2, pp. 169-179, 2000.

[12] H.-H. Yu and J. W. Hutchinson, "Influence of substrate compliance on buckling delamination of thin films," International Journal of Fracture, vol. 113, pp. 39-55, 2002.

[13] M. D. Thouless, "Combined buckling and cracking of films," Journal of the American Ceramic Society, vol. 76, no. 11, pp. 29362938, 1993.

[14] Q. Chen, L. Xu, A. Salo, G. Neto, and G. Freitas, "Reliability study of flexible display module by experiments," in Proceedings of the International Conference on Electronic Packaging Technology \& High Density Packaging (ICEPT-HDP '08), pp. 1-6, Shanghai, July 2008.

[15] W.-H. Chen, H.-C. Cheng, Y.-C. Hsu, R.-H. Uang, and J.-S. $\mathrm{Hsu}$, "Mechanical material characterization of Co nanowires and their nanocomposite," Composites Science and Technology, vol. 68, no. 15-16, pp. 3388-3395, 2008.

[16] C. Y. Cao, Q.-H. Qin, and A. B. Yu, "Micromechanical analysis of heterogeneous composites using hybrid trefftz FEM and hybrid fundamental solution based FEM," Journal of Mechanics, vol. 29, no. 4, pp. 661-674, 2013.

[17] S. J. Hollister, D. P. Fyhrie, K. J. Jepsen, and S. A. Goldstein, "Application of homogenization theory to the study of trabecular bone mechanics," Journal of Biomechanics, vol. 24, no. 9, pp. 825-839, 1991.

[18] R. Hill, "A self-consistent mechanics of composite materials," Journal of the Mechanics and Physics of Solids, vol. 13, no. 4, pp. 213-222, 1965.

[19] G. Taguchi, Taguchi Methods: Signal-to-Noise Ratio for Quality Evaluation, Quality Engineering Series, ASI Press, Trevose, Pa, USA, 1993.

[20] H.-C. Cheng, W.-R. Ciou, W.-H. Chen, J.-L. Kuo, H.-C. Lu, and R.-B. Wu, "Heat dissipation analysis and design of a board-level phased-array transmitter module for $60-\mathrm{GHz}$ communication," Applied Thermal Engineering, vol. 53, no. 1, pp. 78-88, 2013.

[21] W. C. Oliver and G. M. Pharr, "Improved technique for determining hardness and elastic modulus using load and displacement sensing indentation experiments," Journal of Materials Research, vol. 7, no. 6, pp. 1564-1580, 1992. 

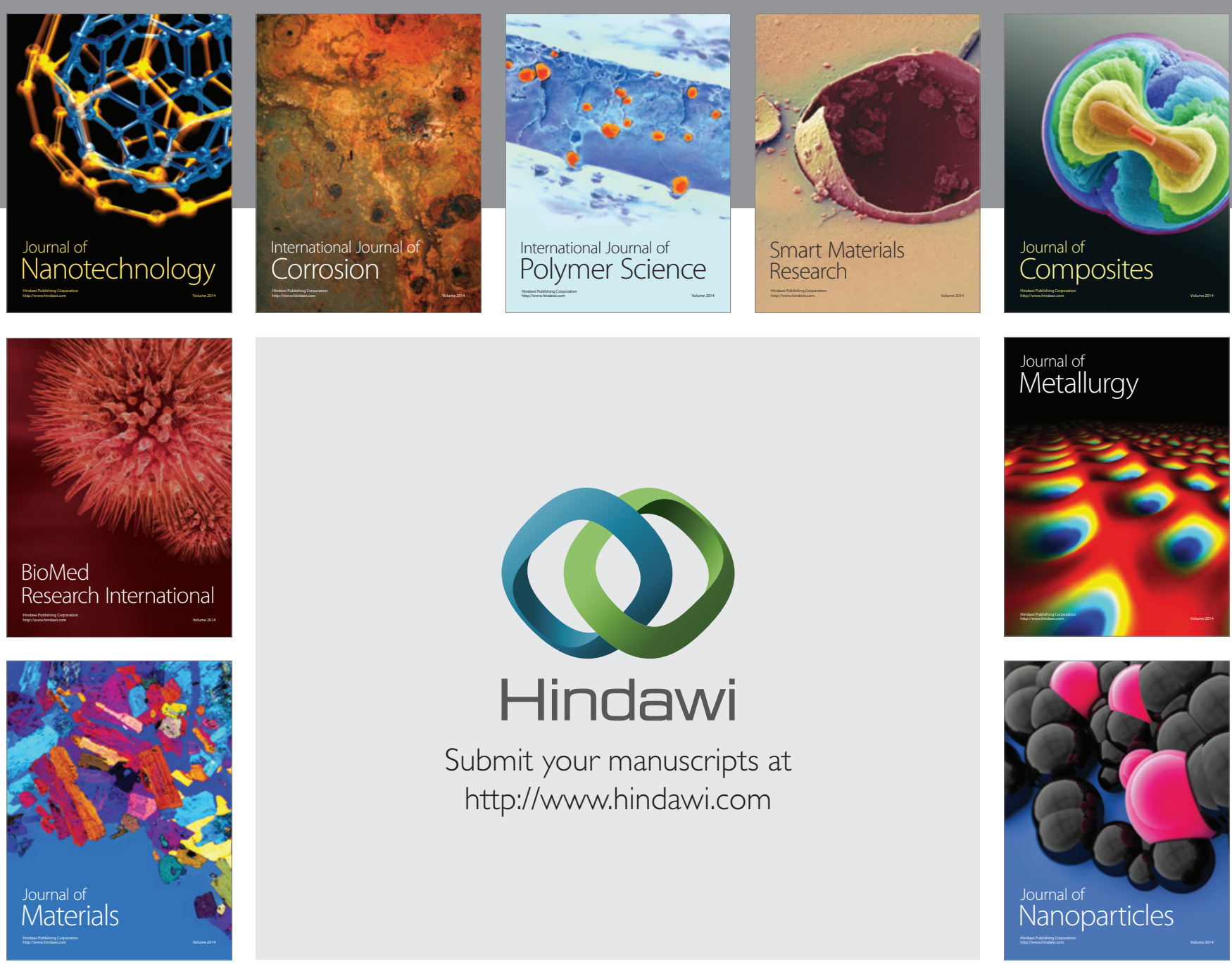

Submit your manuscripts at http://www.hindawi.com
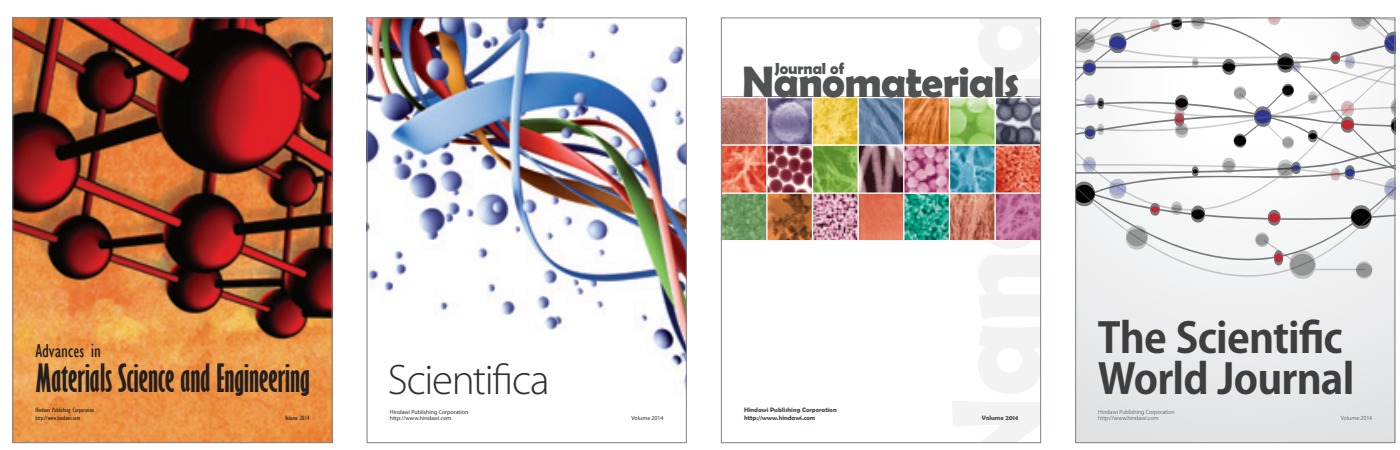

\section{The Scientific World Journal}
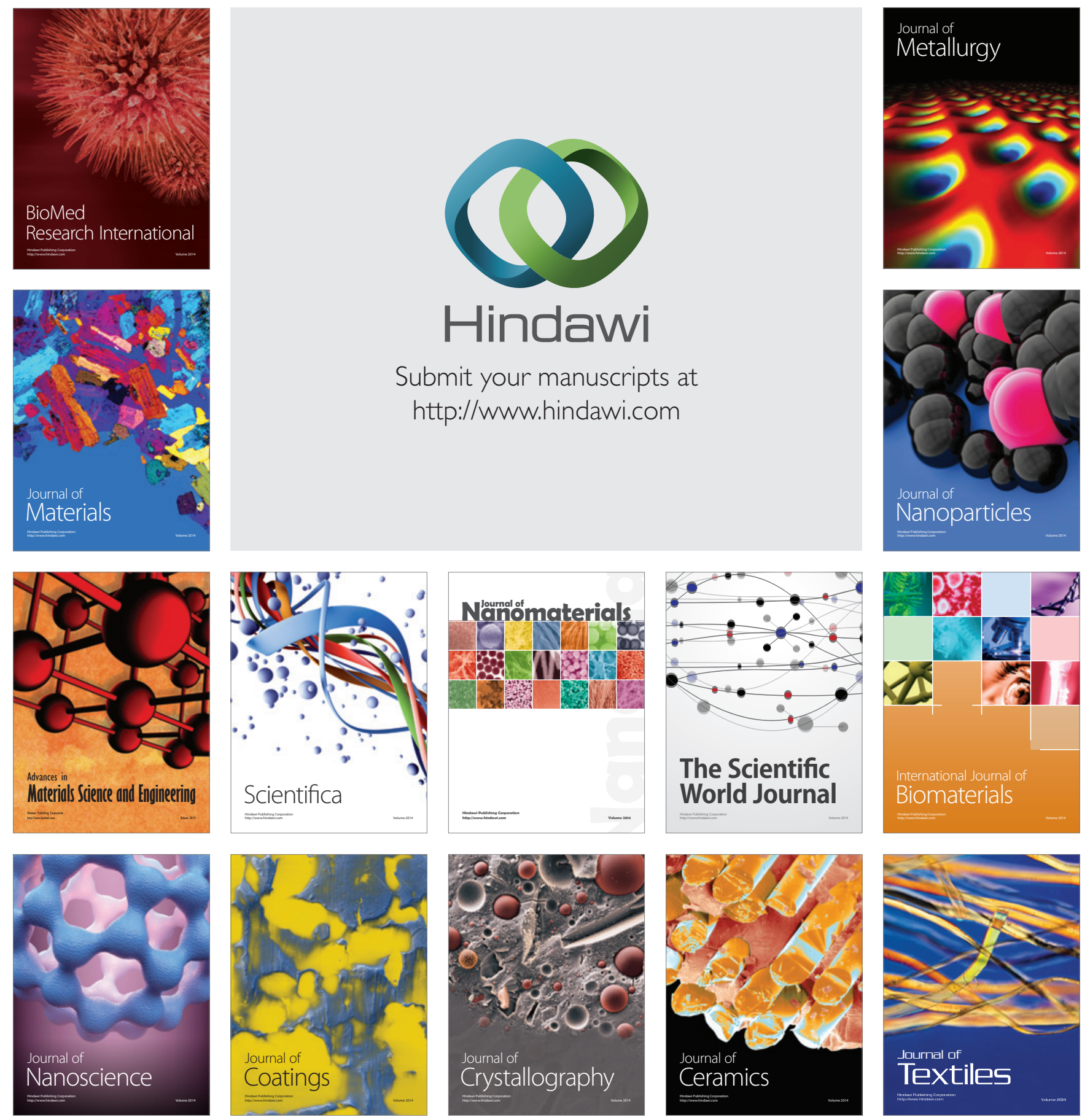